\title{
Dynamical stability of two-dimensional metals in the periodic table
}

\author{
Shota Ono* \\ Department of Electrical, Electronic and Computer Engineering, Gifu University, Gifu 501-1193, Japan
}

\begin{abstract}
We study the dynamical stability of elemental two-dimensional (2D) metals from Li to $\mathrm{Pb}$ by calculating the phonon band structure from first principles, where $2 \mathrm{D}$ structures are assumed to be planer hexagonal, buckled honeycomb, and buckled square lattice structures. We show the relationship between the stability of $2 \mathrm{D}$ structures and that of three-dimensional structures. This provides a material design concept for alloys, where the similarity with regard to the stable 2D structures, rather than the energetic stability of alloy, is important to yield dynamically stable alloys.
\end{abstract}

Most elemental metals have either the face-centered cubic (FCC), hexagonal closed-packed (HCP), or bodycentered cubic (BCC) structures as its ground state [1]. Some elemental metals in a different structure can be dynamically stable, i.e., metastable [2], which are of interest both from a fundamental and practical point of view $[3-7]$. In this paper, we explore dynamically stable structures in two-dimensional (2D) metals and apply them as a building block for computational materials synthesis.

Graphene (C), atomically thin carbon layers, has honeycomb (HC) structure with zero thickness [8]. In contrast, other 2D elements in group 14 have buckled HC (bHC) as a dynamically stable structure $[9,10]$. For example, silicene $(\mathrm{Si})$, germanene $(\mathrm{Ge})$, and stanene $(\mathrm{Sn})$ have a total thickness of $0.45,0.69$, and $0.85 \AA$, respectively [10]. This is attributed to the fact that the bonding in the bulk prefers $s p^{3}$ to $s p^{2}$ character, producing the diamond structure but not the graphite-like structure. Thus far, noble metals $(\mathrm{Cu}$ [11], $\mathrm{Ag}$ [12], and $\mathrm{Au}$ [13]), borophene (B) $[14,15]$ and gallenene $(\mathrm{Ga})[16]$ in group 13, phosphorene (P) [17], arsenene (As) [18], antimonene (Sb) [18], and bithmuthene (Bi) [19] in group 15, poloniumene (Po) in group 16 [20] have been reported as stable 2D materials theoretically and/or experimentally, while B and Ga require external strains (or substrate) to be stabilized. Recently, the stability of elemental 2D metals have been investigated by calculating the total energy within the density-functional theory (DFT) [21, 22]. Although the planer hexagonal (HX) [21] and buckled structures [22] have been proposed as stable structures, no phonon band structure calculations have been performed.

The aim of this paper is to study the dynamical stability of $2 \mathrm{D}$ metals in the periodic table from $\mathrm{Li}$ to $\mathrm{Pb}$ by assuming the planar HX, bHC, and buckled square (bSQ) structures. Figure 1 shows how the stability of $2 \mathrm{D}$ metals is related to that of three-dimensional (3D) metals: (i) If the HX structure is dynamically stable in an elemental metal, the $\mathrm{HCP}$ and/or FCC structures are also stable ( $\mathrm{HX} \rightarrow \mathrm{HCP}$ and/or FCC) except Rb; (ii) If the bHC structure is dynamically stable only, the BCC

*Electronic address: shota_o@gifu-u.ac.jp structure is stable (bHC $\rightarrow \mathrm{BCC}$ ): (iii) If the bSQ structure is dynamically stable only, the HCP structure is stable (bSQ $\rightarrow \mathrm{HCP}$ ) except $\mathrm{Hg}$; and (iv) If the bHC and bSQ structures are dynamically stable, where the bSQ is the metastable phase, the HCP (FCC) structure is stable for group $3,4,7$, and 8 (group 9 and 10) metals (bHC and $\mathrm{bSQ} \rightarrow \mathrm{HCP}$ or FCC). The rest of the paper will be devoted to show this result, to discuss the magnetic effects, and to provide an application to the material design for dynamically stable alloys.

First, we show how the buckled structures become energetically more stable than the planar structure. To demonstrate it, we calculate the total energy of 2D metals based on DFT implemented in Quantum ESPRESSO (QE) code [23]. Spin-polarized calculations are performed for $\mathrm{Cr}, \mathrm{Mn}, \mathrm{Fe}, \mathrm{Co}$, and $\mathrm{Ni}$. The unit cell of the bHC structure includes two atoms, whose positions are given by $(0,0,+\delta)$ and $(0, a / \sqrt{3},-\delta)$ with the lattice constant $a$ and the buckling height $\delta$ (see Fig. 2(b)). If the size of $\delta$ is large enough to satisfy the inequality $(a / \sqrt{3})^{2}+(2 \delta)^{2}>a^{2}$, the bHC can be regarded as the bilayer HX (2HX) structure. Figure 2(a) shows the total energy variation as a function of $\delta$ and $a$ for $2 \mathrm{D} \mathrm{Ag}$, where the total energy is minimum when $\delta=0.0 \AA$ and $a \simeq 4.6 \AA$ and when $\delta \simeq 1.2 \AA$ and $a \simeq 2.8 \AA$. The local minimum of the former and the latter corresponds to the planar $\mathrm{HC}$ and $2 \mathrm{HX}$ structures, respectively. The $2 \mathrm{HX}$ is more stable than the planar HC and HX structures by 0.716 and $0.164 \mathrm{eV} /$ atom, respectively. We also consider the bSQ structure with two atoms included in a unit cell, where the positions of atoms are given by $(0,0,+\delta)$ and $(a / 2, a / 2,-\delta)$, as shown in Fig. $2(\mathrm{~d})$. The bSQ becomes the bilayer SQ (2SQ) structure when the inequality $(a / \sqrt{2})^{2}+(2 \delta)^{2}>a^{2}$ is satisfied. We plot the total energy variation as a function of $\delta$ and $a$ for 2D Ag in Fig. 1(c). The two local minimum correspond to the planar SQ and the 2SQ structures. The values of $a$ giving the minimum total energy are close to the nearest-neighbor (NN) interatomic distance in FCC Ag $(2.89 \AA)$. By setting the initial lattice parameter to the NN interatomic distance in 3D metals [24], we optimize the geometry of the HX, bSQ (or 2SQ), and bHC (or $2 \mathrm{HX}$ ) structures from Li to $\mathrm{Pb}$. More computational details, the cohesive energy, and the structural parameters of these structures are provided in Appendix. 


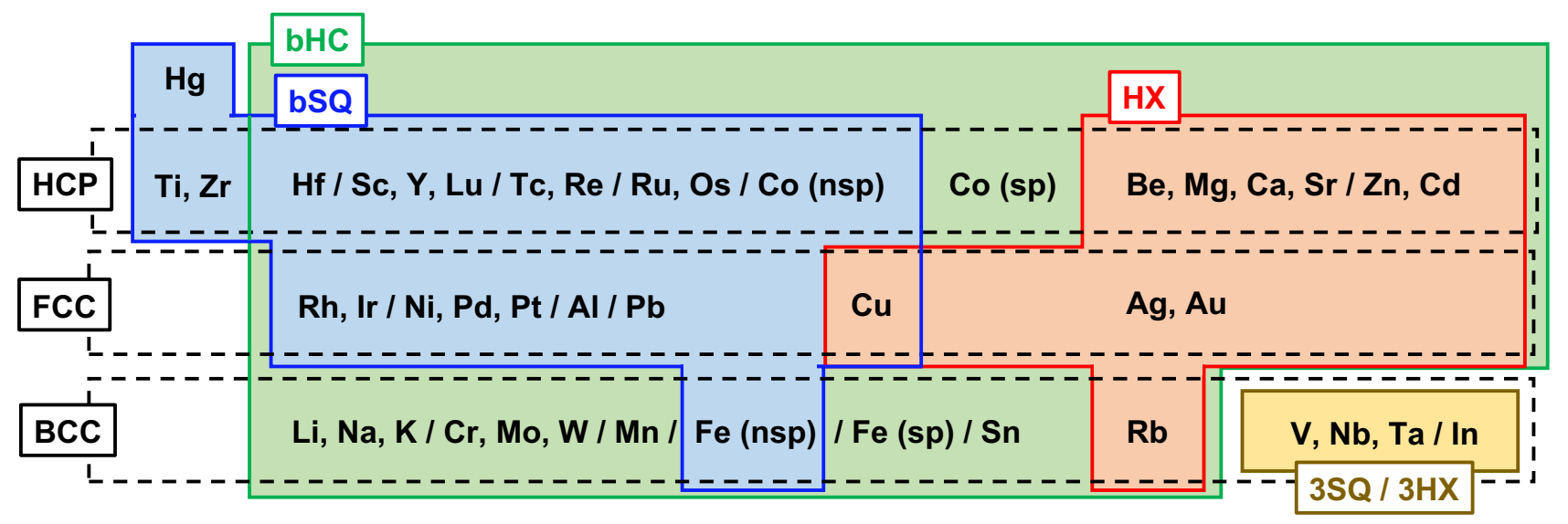

$\mathrm{Cs}, \mathrm{Ba}, \mathrm{TI}, \mathrm{Ga}$ : No stable 2D structures found at zero temperature and pressure.

FIG. 1: Relationship of the dynamical stability between 2D structures (HX, bHC, and bSQ) and 3D structures (HCP, FCC, and BCC) in elemental metals. The stability of multilayered structures (3SQ and 3HX) is investigated if the HX, bHC, and bSQ structures are unstable. The stability property of spin-polarized (sp) Co and Fe is different from that of non-spin-polarized (nsp) ones. Stable crystal structures in 3D metals are extracted from the periodic table depicted in Ref. [1]. The cubic Mn and tetragonal In and $\mathrm{Sn}$ are included to the group of BCC metals. $\mathrm{Hg}$ has the rhombohedral structure as its ground state [1]. Note that 2D Ga becomes dynamically stable when strains are imposed [16].

(a)

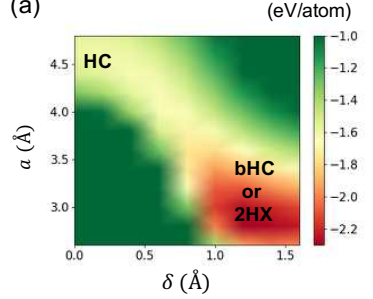

(c)

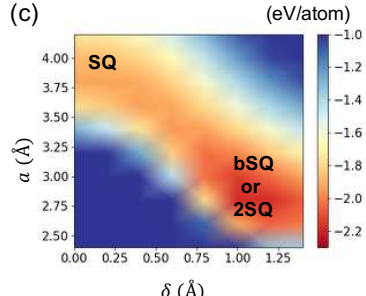

(b)

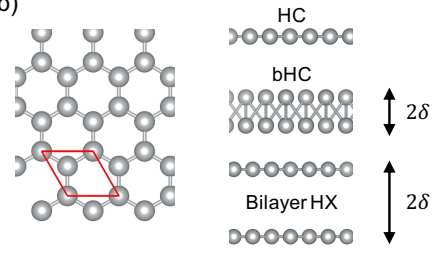

(d)

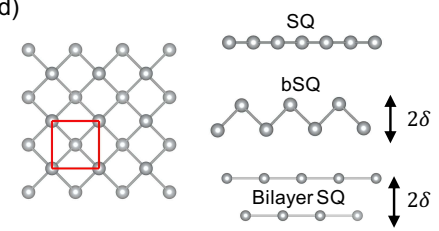

FIG. 2: (a) The total energy variation as a function of $a$ and $\delta$ and (b) top and side views for the bHC (or 2HX) structures of Ag. (c) and (d): Same as (a) and (b), respectively, but for the bSQ (or 2SQ) structure. The unit cell is enclosed by red lines. The layer thickness (or the interlayer distance in the bilayer structure) is equal to $2 \delta$.

Next, we study the dynamical stability of the optimized geometries by performing the phonon band structure calculations within the density-functional perturbation theory (DFPT) [25] implemented in QE code [23]. The phonon energy is defined by $E_{p}=\operatorname{sgn}\left(\omega^{2}\right) \hbar|\omega|$, where sgn is the sign function, $\hbar$ is the Planck constant, and $\omega$ is the phonon frequency obtained by diagonalizing the dynamical matrix. $E_{p}$ is negative when $\omega$ is an imaginary number.

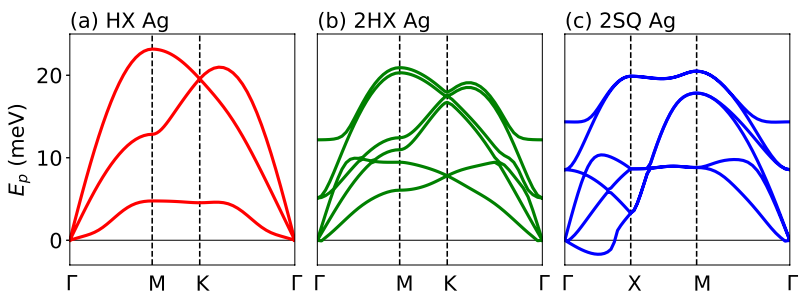

FIG. 3: Phonon band structure of $\mathrm{Ag}$ in (a) HX, (b) 2HX, and (c) 2SQ structures.

Figure 3 shows the phonon band structure of Ag. The HX and 2HX structures are dynamically stable: No imaginary frequencies are observed. The former case is consistent with the previous calculations [12]. However, the 2SQ structure is unstable because the imaginary frequency appears along $\Gamma$-X line. Note that the $2 \mathrm{SQ}$ is energetically more stable than the planar HX structure by $0.057 \mathrm{eV} /$ atom. This implies that the large cohesive energy does not always lead to the dynamical stability, as has been observed in many alloys [26], while it can lead to an increase in the flexural phonon energy particularly at the point $\mathrm{M}$. The computational details and the phonon band structures of other elements are provided in Appendix. Below, we will not distinguish the bHC (bSQ) and $2 \mathrm{HX}$ (2SQ) structures and use the "bHC" and "bSQ" only.

Stable 2D structures. We begin with $\mathrm{Zn}$ and $\mathrm{Cd}$ that have the HX structure. It is well known that these have HCP structure, in which the ratio of the lattice constants $(c / a=1.86$ and 1.89 for $\mathrm{Zn}$ and $\mathrm{Cd}$, respectively) is much larger than the ideal value $c / a=1.63$ [1]. It is reason- 
able that they can be exfoliated easily and have monolayer HX structure. The alkali earth (Be, Mg, Ca, and $\mathrm{Sr})$ and noble metals $(\mathrm{Cu}, \mathrm{Ag}$, and $\mathrm{Au})$ that are classified into divalent and monovalent metals having two and one $s$ electrons in the outermost shell, respectively. Since the distribution of the $s$ electron is spherically symmetric around the nucleus, the densely packed HX structure with no buckling is realized. In this sense, it is strange that the HX structure is unstable in alkali metals $(\mathrm{Li}$, $\mathrm{Na}$, and $\mathrm{K}$ ).

All transition metals have buckled or multilayered structures. For these metals, the electronic states around the Fermi level consist of a mixture of $s$ and $p(d)$ electrons. Such a hybridized orbital is less spherical symmetry, creating the buckled structures, as realized in group 14 semiconductors ( $\mathrm{Si}$ and $\mathrm{Ge}$ ) [9]. With respect to the stability, the trivalent metal of $\mathrm{Al}$ is similar to transition metals rather than free-electron metals ( $\mathrm{Na}$ and $\mathrm{K}$ ). It is interesting to note that in $\mathrm{Cr}, \mathrm{Hg}, \mathrm{Mo}, \mathrm{Nb}, \mathrm{Ti}, \mathrm{V}, \mathrm{W}$, and $\mathrm{Zr}$, the cohesive energy of bSQ is larger than that of bHC, although the energetic stability is not necessary to produce the dynamical stability of the bSQ structure.

In case that the $\mathrm{HX}, \mathrm{bHC}$, and bSQ structures are unstable, we examine the dynamical stability of the trilayer structures. The BCC V, Nb, and Ta are dynamically stable when trilayer SQ (3SQ) structure is assumed, whereas the tetragonal In is dynamically stable in trilayer HX $(3 \mathrm{HX})$ structure, where the positions of atoms in a unit cell are $(0,0,0)$, and $(a / 2, a / 2, \pm 2 \delta)$ for 3SQ and $(0,0,0)$, and $(0, a / \sqrt{3}, \pm 2 \delta)$ for $3 \mathrm{HX}$ structures. Unfortunately, no dynamically stable structures are found for $\mathrm{Cs}, \mathrm{Ba}, \mathrm{Ga}$, and $\mathrm{Tl}$ at zero pressure. The instability of these elements are due to the instability against the out-of-plane (ZA) vibrations. Fortunately, the imaginary frequencies appear around $\Gamma$ point only, meaning that these $2 \mathrm{D}$ metals would be stable when the lateral size of the sample is smaller than the wavelength of the corresponding ZA phonons.

Magnetic effects. Through analyses of the magnetic moment in period-4 metals, we find that the behavior of $\mathrm{bHC}$ is quite analogous to that of HCP phase. The bHC phase of $\mathrm{Cr}, \mathrm{Mn}$, and $\mathrm{Fe}$ has nonmagnetic, while that of $\mathrm{Co}$ and Ni has ferromagnetic state with the magnetic moment 1.89 and $0.82 \mu_{\mathrm{B}}$ per atom, respectively (the Bohr magneton $\left.\mu_{\mathrm{B}}\right)$. For $3 \mathrm{D}$ case, the $\mathrm{HCP}$ phase of $\mathrm{Mn}$ and $\mathrm{Fe}$ has nonmagnetic, while that of $\mathrm{Cr}$, $\mathrm{Co}$, and $\mathrm{Ni}$ has ferromagnetic state with $0.12,1.60$, and $0.59 \mu_{\mathrm{B}}$ per atom, respectively [27]. Within non-spin-polarized calculations, bHC Co and $\mathrm{Ni}$ are also dynamically stable. This is reasonable because HCP Co and Ni are elastically stable in the absence of magnetic effects [28].

The trend of the dynamical stability in bSQ is different from that of bHC structure. Figure 4 shows the phonon band structure of bSQ Fe, Co, and Ni: Left and right for the non-spin-polarized and spin-polarized calculations, respectively. Within non-spin-polarized calculations, the bSQ structure is dynamically stable. However, except $\mathrm{Ni}$, those structures become dynamically unstable
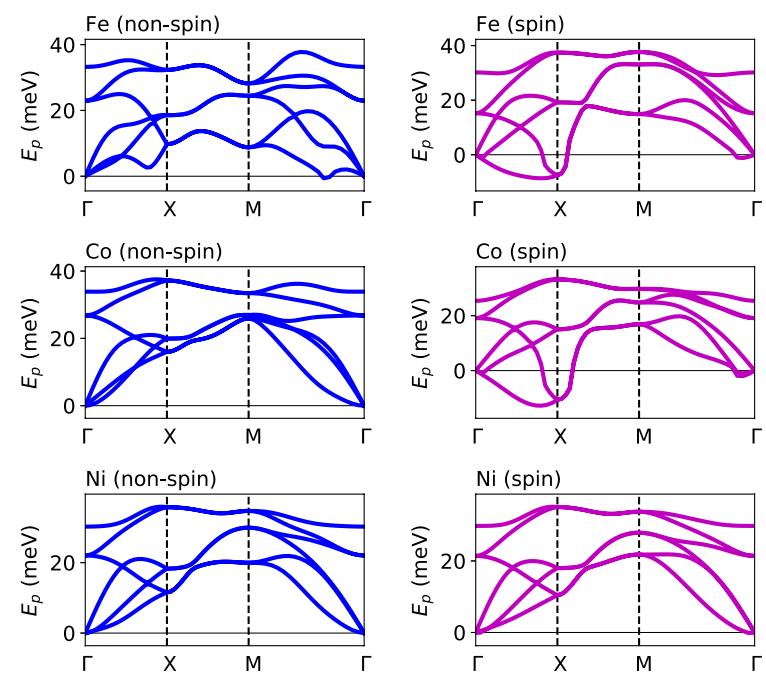

FIG. 4: The phonon band structure of Fe, Co, and Ni that have bSQ structure. Left for non-spin-polarized and right for spin-polarized calculations.

when magnetic effects are included, where the absolute magnetic moment per atom is $2.77,1.94$, and $0.82 \mu_{B}$ for ferromagnetic $\mathrm{Fe}, \mathrm{Co}$, and $\mathrm{Ni}$, respectively. This trend is similar to the stability of FCC Fe, where it is elastically stable and unstable in nonmagnetic and ferromagnetic states, respectively [28].

Material design principles. We discuss how the dynamical stability of the $2 \mathrm{D}$ structure is related to that of the $3 \mathrm{D}$ structure in elemental metals. The property (i), i.e., $\mathrm{HX} \rightarrow \mathrm{HCP}$ and/or FCC, can be derived from the fact that the FCC and HCP structures consist of HX monolayers stacked along the (111) direction and the $c$ axis, respectively [1]. The property (ii), i.e., bHC or trilayer $\rightarrow \mathrm{BCC}$, is also a reasonable conclusion because the BCC structure is obtained by stacking the bHC and 3SQ structures along the (111) direction and $c$ axis, respectively. The property (iii), i.e., bSQ $\rightarrow \mathrm{HCP}$, is derived from the fact that the HCP structure is obtained by stacking the elongated bSQ (along the diagonal direction of the square shaped unit cell) along the $c$ axis. The property (iv), i.e., bHC and $\mathrm{bSQ} \rightarrow \mathrm{HCP}$ or $\mathrm{FCC}$, may be understood as in the properties of (i) and (iii), while it is difficult to disentangle the stability properties of FCC and HCP. The understanding of the metastability of bSQ phase will be a key to resolve this issue.

We propose that the stability relationship between $2 \mathrm{D}$ and 3D metals can be applied to a prediction of the dynamical stability of 3D alloys. In the present study, we focus on the binary $\mathrm{Al}-\mathrm{Cu}$ system that has a complex phase diagram [29]. For the case of $\mathrm{Al}_{0.5} \mathrm{Cu}_{0.5}$, it is natural to consider that $\mathrm{AlCu}$ has the $\mathrm{B}_{h}$ (WC) structure with $\mathrm{HX} \mathrm{Al}$ and $\mathrm{HX} \mathrm{Cu}$ layers stacked alternately, as shown in Fig. 5(a), since the bHC and HX structures in 

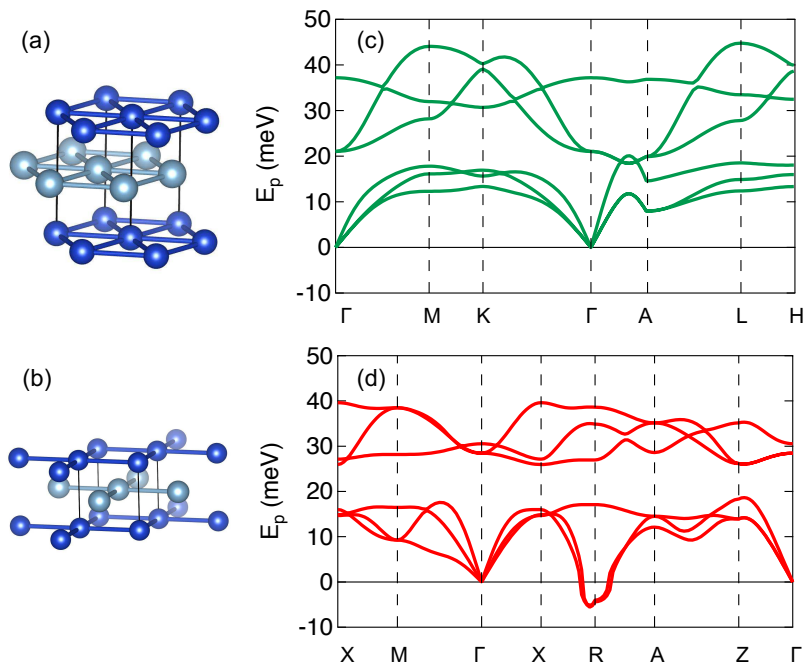

FIG. 5: Schematic illustration of (a) $\mathrm{B}_{h}$ and (b) $\mathrm{L} 1_{0}$ structures. The phonon band structure of (c) $\mathrm{B}_{h}$ and (d) $\mathrm{L} 1_{0} \mathrm{AlCu}$ alloys.

$\mathrm{Al}$ and $\mathrm{Cu}$ are dynamically stable, respectively, as shown in Fig. 1. Meanwhile, the $\mathrm{L}_{0}(\mathrm{CuAu})$ structure (see Fig. 5(b)) has been predicted to be more energetically stable structure [30]. This may be reasonable because $\mathrm{Al}$ and $\mathrm{Cu}$ have the bSQ as a metastable structure and also because the $\mathrm{L}_{0}$ structure consists of the SQ lattice of $\mathrm{Al}$ and the displaced $\mathrm{SQ}$ lattice of $\mathrm{Cu}$ stacked alternately along $c$ axis. Figures $5(\mathrm{c})$ and $5(\mathrm{~d})$ show the phonon band structure of $\mathrm{AlCu}$ alloy in the $\mathrm{B}_{h}$ and $\mathrm{L} 1_{0}$ structures, respectively, indicating that the $\mathrm{B}_{h}$ is dynamically stable, whereas the $\mathrm{L} 1_{0}$ is unstable. The stability of the $\mathrm{B}_{h}$ phase is robust against the thermal fluctuations up to $2500 \mathrm{~K}$, which is confirmed by performing $a b$ initio molecular dynamics (MD) simulations implemented in QE code [23]; see Appendix for the computational details. We again emphasize that the energetic stability is not enough to yield the dynamical stability in alloys; For the present case, the $\mathrm{L} 1_{0}$ structure is more stable than the $\mathrm{B}_{h}$ structure by $0.058 \mathrm{eV}$ per unit cell. We can demonstrate that $\mathrm{B}_{h} \mathrm{CuZn}$ is dynamically stable, while CuZn has B2 ( $\mathrm{CsCl})$ structure as its ground state [31] as provided in Appendix. Our approach based on the list of dynamically stable structures in Fig. 1 provides a novel principle for computational design of alloys.

Future perspective. In order to establish a design principle for alloys, it would be important to understand the origin of metastability in both elemental metals and alloys $[3,26]$ and to calculate the energy landscape as a function of atom positions [32] in alloys. It is interesting to discuss how the present approach is related to the Pettifor's approach for the stability of binary alloys [33]. The present study is limited to the phonons of free-standing and ideal thin films of elemental metals at zero temperature and pressure. It would be interesting to study how the effects of substrate (or strain) [16], chemical adsorption [22], vacancies [34], grain boundaries [35], and temperature [36] influence the phonon band structure and the dynamical stability of $2 \mathrm{D}$ metals. We note that the effect of spin-orbit coupling is important in heavy elements because it strongly influences the dynamical stability of 2D Bi [19] and 2D Po [20] as well as the metastability of FCC Pt in the HCP structure [4]. For fundamental interest, it is interesting to calculate the electron-phonon coupling function (Eliashberg function) of 2D metals in order to find novel 2D superconductors [37-40]. It is also interesting to explore whether $2 \mathrm{D} \mathrm{Mn}$ has more complex structures, since 3D Mn has a BCC-related structure with 58 atoms per unit cell [41].

\section{Acknowledgments}

This study is supported by the Nikki-Saneyoshi Foundation. A part of numerical calculations has been done using the facilities of the Supercomputer Center, the Institute for Solid State Physics, the University of Tokyo.

\section{Appendix A: Computational details}

We calculate the total energy of $2 \mathrm{D}$ metals based on DFT implemented in Quantum ESPRESSO (QE) code [23]. The effects of exchange and correlation are treated within GGA-PBE [42] and GGA-PBEsol [43]. We use the ultrasoft pseudopotentials generated by the scheme of Ref. [44], i.e., pslibrary.1.0.0. For Na, $\mathrm{Mn}$, and Tc, pslibrary.0.2, pslibrary.0.3.1, and pslibrary.0.3.0 are used, respectively. The cutoff energies for the wavefunction and the charge density are 80 Ry and 800 Ry, respectively, which are well above the suggested cutoff energies. The self-consistent calculations are performed by using $30 \times 30 \times 1 k$ grid for $2 \mathrm{D}$ metals and $15 \times 15 \times 15 k$ grid for $3 \mathrm{D}$ alloys [45]. The primitive lattice vectors in units of the lattice constant $a$ are $(1,0,0),(-1 / 2, \sqrt{3} / 2,0)$, and $(0,0, c / a)$ for HX, $2 \mathrm{HX}$, and $3 \mathrm{HX}$ structures, and $(1,0,0),(0,1,0)$, and $(0,0, c / a)$ for 2SQ and 3SQ structures. The size of $c$ is fixed to be $14 \AA$. The Marzari-Vanderbilt smearing [46] with a broadening of $\sigma=0.02 \mathrm{Ry}$ is used for all calculations.

The phonon band structure calculations are performed within the density-functional perturbation theory [25] implemented in QE code [23]. For HX and bHC structures, $8 \times 8 \times 1 q$ grid ( $10 q$ points) is used, while for bSQ structure, $6 \times 6 \times 1 q$ grid (10 $q$ points) is used. For $3 \mathrm{HX}$ and $3 \mathrm{SQ}$ structures, $6 \times 6 \times 1 q$ grid $(7$ and $10 q$ points, respectively) is used. In cases of $\mathrm{bHC} \mathrm{W}$, bSQ W, and anti-ferromagnetic phase of $\mathrm{HX} \mathrm{Cr}$ and $\mathrm{Mn}$, the size of $q$ grid is decreased to $6 \times 6 \times 1,4 \times 4 \times 1$, and $4 \times 2 \times 1$, respectively, in order to reduce the computational costs. For $\mathrm{AlCu} \mathrm{CuZn}$ alloys, $4 \times 4 \times 4 q$ grid is used.

The ab initio molecular dynamics (MD) simulations for $\mathrm{B}_{h} \mathrm{AlCu}$ and $\mathrm{CuZn}$ alloys are performed using $\mathrm{QE}$ code 


\begin{tabular}{|c|c|c|c|c|c|c|c|c|c|c|c|c|c|c|c|c|}
\hline & 1 & 2 & 3 & 4 & 5 & 6 & 7 & 8 & 9 & 10 & 11 & 12 & 13 & 14 & 15 & 16 \\
\hline 2 & $\begin{array}{c}\mathrm{Li} \\
\mathrm{bHC} \\
- \\
\mathrm{BCC}\end{array}$ & $\begin{array}{c}\mathrm{Be} \\
\mathrm{HX} \\
- \\
\mathrm{HCP}\end{array}$ & \multicolumn{3}{|c|}{$\begin{array}{c}\text { Symbol } \\
\text { 2D HX structure } \\
\text { 2D SQ structure } \\
\text { 3D structure }\end{array}$} & \multirow{2}{*}{\multicolumn{3}{|c|}{$\begin{array}{c}\mathrm{HX}=\text { hexagonal } \\
\mathrm{bHC}=\text { buckled honeycomb } \\
3 \mathrm{HX}=3 \text {-layered hexagonal } \\
\mathrm{bSQ}=\text { buckled square } \\
3 \mathrm{SQ}=3 \text {-layered square } \\
\mathrm{FCC}=\text { face-centered cubic } \\
\mathrm{BCC}=\text { body-centered cubic } \\
\mathrm{SC}=\text { simple cubic }\end{array}$}} & \multirow{2}{*}{\multicolumn{3}{|c|}{$\begin{array}{c}\text { CUB }=\text { cubic } \\
\text { TET }=\text { tetragonal } \\
\text { ORC }=\text { orthorhombic } \\
\text { HCP }=\text { hexagonal closed } \\
\text { packed } \\
\text { DIA = diamond } \\
\mathrm{RHL}=\text { rhombohedral }\end{array}$}} & & $\begin{array}{c}\text { B } \\
\text { stripe } \\
\text { TET }\end{array}$ & $\begin{array}{c}\text { C } \\
\text { HC } \\
\text { graphite }\end{array}$ & & \\
\hline 3 & $\begin{array}{c}\mathrm{Na} \\
\mathrm{bHC} \\
- \\
\mathrm{BCC}\end{array}$ & $\begin{array}{c}\mathrm{Mg} \\
\mathrm{HX} \\
- \\
\mathrm{HCP}\end{array}$ & & & & & & & & & & & $\begin{array}{c}\mathrm{Al} \\
\mathrm{bHX} \\
(\mathrm{bSQ}) \\
\mathrm{FCC}\end{array}$ & $\begin{array}{c}\mathbf{S i} \\
\mathrm{bHC} \\
\mathrm{DIA}\end{array}$ & $\begin{array}{l}\stackrel{P}{b H C} \\
\text { CUB }\end{array}$ & \\
\hline 4 & $\begin{array}{c}\mathbf{K} \\
\mathrm{bHC} \\
- \\
\mathrm{BCC}\end{array}$ & $\begin{array}{c}\mathrm{Ca} \\
\mathrm{HX} \\
- \\
\mathrm{FCC}\end{array}$ & $\begin{array}{c}\text { Sc } \\
\text { bHC } \\
\text { (bSQ) } \\
\text { HCP }\end{array}$ & $\begin{array}{c}\mathrm{Ti} \\
- \\
\mathrm{bSQ} \\
\mathrm{HCP}\end{array}$ & $\begin{array}{c}\mathbf{v} \\
- \\
3 S Q \\
B C C\end{array}$ & $\begin{array}{c}\mathrm{Cr} \\
\mathrm{bHC} \\
- \\
\mathrm{BCC}\end{array}$ & $\begin{array}{c}\mathrm{Mn} \\
\mathrm{bHC} \\
- \\
\text { CUB }\end{array}$ & $\begin{array}{c}\mathrm{Fe} \\
\mathrm{bHC} \\
- \\
\mathrm{BCC}\end{array}$ & $\begin{array}{c}\text { Co } \\
\text { bHC } \\
- \\
\text { HCP }\end{array}$ & $\begin{array}{c}\mathbf{N i} \\
\mathrm{bHX} \\
(\mathrm{bSQ}) \\
\mathrm{FCC}\end{array}$ & $\begin{array}{c}\mathrm{Cu} \\
\mathrm{HX} \\
\text { (bSQ) } \\
\text { FCC }\end{array}$ & $\begin{array}{c}\mathrm{Zn} \\
\mathrm{HX} \\
- \\
\mathrm{HCP}\end{array}$ & $\begin{array}{c}\text { Ga } \\
- \\
- \\
\text { ORC }\end{array}$ & $\begin{array}{c}\mathrm{Ge} \\
\mathrm{bHC} \\
\mathrm{DIA}\end{array}$ & $\begin{array}{c}\text { As } \\
\text { bHC } \\
\text { RHL }\end{array}$ & \\
\hline 5 & $\begin{array}{c}\mathbf{R b} \\
\mathrm{HX} \\
- \\
\mathrm{BCC}\end{array}$ & $\begin{array}{c}\mathrm{Sr} \\
\mathrm{HX} \\
- \\
\mathrm{FCC}\end{array}$ & $\begin{array}{c}\mathbf{Y} \\
\mathrm{bHC} \\
(\mathrm{bSQ}) \\
\mathrm{HCP}\end{array}$ & $\begin{array}{c}\mathrm{Zr} \\
- \\
\mathrm{bSQ} \\
\mathrm{HCP}\end{array}$ & $\begin{array}{c}\mathrm{Nb} \\
- \\
3 \mathrm{SQ} \\
\mathrm{BCC}\end{array}$ & $\begin{array}{c}\text { Mo } \\
\mathrm{bHC} \\
- \\
\mathrm{BCC}\end{array}$ & $\begin{array}{c}\mathrm{Tc} \\
\mathrm{bHC} \\
\text { (bSQ) } \\
\mathrm{HCP}\end{array}$ & $\begin{array}{c}\mathrm{Ru} \\
\mathrm{bHC} \\
\text { (bSQ) } \\
\mathrm{HCP}\end{array}$ & $\begin{array}{c}\mathbf{R h} \\
\text { bHX } \\
(\mathrm{bSQ}) \\
\text { FCC }\end{array}$ & $\begin{array}{c}\text { Pd } \\
\text { bHX } \\
(\mathrm{bSQ}) \\
\mathrm{FCC}\end{array}$ & $\begin{array}{c}\mathrm{Ag} \\
\mathrm{HX} \\
- \\
\mathrm{FCC}\end{array}$ & $\begin{array}{c}\mathrm{Cd} \\
\mathrm{HX} \\
- \\
\mathrm{HCP}\end{array}$ & $\begin{array}{c}\text { In } \\
3 \mathrm{HX} \\
- \\
\text { TET }\end{array}$ & $\begin{array}{c}\text { Sn } \\
\text { bHC } \\
- \\
\text { TET }\end{array}$ & $\begin{array}{c}\mathrm{Sb} \\
\mathrm{bHC} \\
\mathrm{RHL}\end{array}$ & \\
\hline 6 & $\begin{array}{c}\text { Cs } \\
- \\
- \\
B C C\end{array}$ & $\begin{array}{c}\mathrm{Ba} \\
- \\
- \\
\mathrm{BCC}\end{array}$ & $\begin{array}{c}\text { Lu } \\
\text { bHC } \\
\text { (bSQ) } \\
\text { HCP }\end{array}$ & $\begin{array}{c}\text { Hf } \\
\text { bHC } \\
\text { (bSQ) } \\
\text { HCP }\end{array}$ & $\begin{array}{c}\text { Ta } \\
- \\
3 S Q \\
B C C\end{array}$ & $\begin{array}{c}\mathbf{w} \\
\mathrm{bHC} \\
- \\
\mathrm{BCC}\end{array}$ & $\begin{array}{c}\mathbf{R e} \\
\text { bHC } \\
\text { (bSQ) } \\
\text { HCP }\end{array}$ & $\begin{array}{c}\text { Os } \\
\text { bHC } \\
\text { (bSQ) } \\
\text { HCP }\end{array}$ & $\begin{array}{c}\text { Ir } \\
\text { bHX } \\
\text { (bSQ) } \\
\text { FCC }\end{array}$ & $\begin{array}{c}\text { Pt } \\
\text { bHX } \\
\text { (bSQ) } \\
\text { FCC }\end{array}$ & $\begin{array}{c}\mathrm{Au} \\
\mathrm{HX} \\
- \\
\mathrm{FCC}\end{array}$ & $\begin{array}{c}\mathbf{H g} \\
- \\
\mathrm{bSQ} \\
\mathrm{RHL}\end{array}$ & $\begin{array}{c}\mathrm{TI} \\
- \\
- \\
\mathrm{HCP}\end{array}$ & $\begin{array}{c}\mathrm{Pb} \\
\mathrm{bHC} \\
\text { (bSQ) } \\
\mathrm{FCC}\end{array}$ & $\begin{array}{c}\mathrm{Bi} \\
\mathrm{bHC} \\
\mathrm{RHL}\end{array}$ & $\begin{array}{l}\text { Po } \\
\text { SQ } \\
\text { SC }\end{array}$ \\
\hline & & & & & & & (iii) & $S Q$ & & $\begin{array}{r}\text { (iv) } 3 \\
3\end{array}$ & $\begin{array}{l}\mathrm{X} X \text { or } \\
\mathrm{Q}\end{array}$ & & $\begin{array}{l}(v) N \\
\text { zerot }\end{array}$ & רpera & & \\
\hline
\end{tabular}

FIG. 6: Periodic table for elemental 2D materials. The present results are colored. The stable 2D structures are indicated (The structure in parenthesis indicates the metastable phase). Stable crystal structures in 3D materials are from Ref. [1]. The previously investigated structures that have dynamical stability include semiconductors of C [8], Si, Ge, and Sn [10], P [17], and $\mathrm{As}$ and $\mathrm{Sb}[18]$, and metals of $\mathrm{Cu}[11], \mathrm{Ag}[12], \mathrm{Au}[13], \mathrm{B}[14,15], \mathrm{Ga}[16], \mathrm{Bi}[19]$, and $\mathrm{Po}$ [20].

[23]. The ionic temperature is controlled via the velocity scaling and increased from $500 \mathrm{~K}$ up to $3000 \mathrm{~K}$ with an increment of $500 \mathrm{~K}$. A $2 \times 2 \times 2$ supercell with 16 atoms is considered. The Newton's equation is integrated by using Verlet algorithm with a time step of $0.967 \mathrm{fs}(20$ a.u.) and up to $2.0 \mathrm{ps}$ (2100 MD steps).

Throughout the main text and this supplemental material, we discuss the dynamical stability of $2 \mathrm{D}$ metals and 3D alloys based on the GGA-PBE calculation results. It would be valuable to note that the dynamically stable structures obtained from the GGA-PBEsol calculations are almost the same as those obtained from the GGA-PBE calculations. The differences are as follows: Within GGA-PBEsol, HX K, bSQ Hg, and 3HX Tl are dynamically stable, while bSQ $\mathrm{Cu}$ and $\mathrm{HX}$ Au are unstable. We have not studied the dynamical stability of $\mathrm{Nb}$ and Tc because no GGA-PBEsol potentials are available.

\section{Appendix B: Structural parameters}

The cohesive energy of the structure $j$ is defined as $E_{\mathrm{c}, j}=E_{\text {atom }}-E_{j}$, where $E_{\text {atom }}$ is the total energy of an atom in free space and $E_{j}$ is the total energy per atom of the structure $j$. During the geometry optimization for the structure $j$, the total energy and forces are converged within $10^{-5}$ Ry and $10^{-4}$ a.u., respectively. The value of $E_{\text {atom }}$ for all atoms is obtained from atom-in-a-box calculations (within non-spin-polarized approximation) of a single atom in a unit cell with a volume of $15 \times 15 \times 15$ $\AA^{3}$. Table I lists $E_{\mathrm{c}, j}$, the lattice constant $a_{j}$, and the buckling height $\delta_{j}$, with the subscripts $j=1$ for the HX, $j=2$ for the bHC or $2 \mathrm{HC}$, and $j=3$ for the bSQ or $2 \mathrm{SQ}$ structures, where the data of $\delta_{1}$ that is equal to zero is omitted.

For 3SQ V, 3SQ Nb, 3SQ Ta, and 3HX In, the cohesive energy $E_{\mathrm{c}, 4}(\mathrm{eV})$, the lattice constant $a_{4}(\AA)$, and the interlayer distance $2 \delta_{4}(\AA)$ are $\left(E_{\mathrm{c}, 4}, a_{4}, 2 \delta_{4}\right)=$ (6.947, 2.886, 1.404), (8.209, 3.256, 1.471), (8.195, 3.258, $1.469)$, and $(2.223,3.315,2.768)$, respectively.

For $\mathrm{AlCu}$ alloys, the optimized lattice constants $(a, c)$ are $(2.787,4.077) \AA$ for $\mathrm{B}_{h}$ structure $(c / a=1.463)$ and $(2.900,3.202) \AA$ for $\mathrm{L}_{0}$ structure $(c / a=1.104)$. For $\mathrm{B}_{h}$ $\mathrm{CuZn}$, the lattice parameters $(a, c)$ are $(2.676,4.211) \AA$, i.e., $c / a=1.574$.

\section{Appendix C: Phonon band structures}

Figures 7-52 show the phonon band structures from $\mathrm{Li}$ to $\mathrm{Pb}$ for (a) HX, (b) bHC, and (c) bSQ structures. The phonon band structure and the trend of the dynamical stability of HX, bHC, and bSQ are similar in the same group in the periodic table. Below, the results for non-spin-polarized calculations are shown. The phonon band structures of bHC Co (spin-polarized), bHC $\mathrm{Ni}$ (spin-polarized), trilayer $\mathrm{V}, \mathrm{Nb}, \mathrm{Ta}, \mathrm{Ga}, \mathrm{In}$, and $\mathrm{Tl}$ will be shown at the end of SM. The stability properties of elemental metals are summarized in the periodic table shown in Fig. 6. 
TABLE I: $E_{\mathrm{c}, j}(\mathrm{eV} /$ atom $), a_{j}(\AA)$, and $\delta_{j}(\AA)$ for $j=1,2,3$. "nsp" and "sp" in the parenthesis indicate the non-spin-polarized and spin-polarized calculations, respectively.

\begin{tabular}{|c|c|c|c|c|c|c|c|c|}
\hline & $E_{\mathrm{c}, 1}$ & $a_{1}$ & $E_{\mathrm{c}, 2}$ & $a_{2}$ & $\delta_{2}$ & $E_{\mathrm{c}, 3}$ & $a_{3}$ & $\delta_{3}$ \\
\hline $\mathrm{Ag}$ & 2.144 & 2.794 & 2.307 & 2.855 & 1.239 & 2.201 & 2.802 & 1.082 \\
\hline $\mathrm{Al}$ & 2.842 & 2.682 & 3.217 & 2.748 & 1.227 & 3.085 & 2.713 & 1.088 \\
\hline $\mathrm{Au}$ & 2.845 & 2.748 & 2.897 & 2.773 & 1.442 & 2.722 & 2.756 & 1.143 \\
\hline $\mathrm{Ba}$ & 1.203 & 4.467 & 1.593 & 4.493 & 1.763 & 1.547 & 4.339 & 1.609 \\
\hline $\mathrm{Be}$ & 2.997 & 2.126 & 3.354 & 2.157 & 0.959 & 3.181 & 2.090 & 0.799 \\
\hline $\mathrm{Ca}$ & 1.181 & 3.866 & 1.584 & 3.924 & 1.503 & 1.530 & 3.792 & 1.340 \\
\hline $\mathrm{Cd}$ & 0.487 & 2.922 & 0.642 & 2.950 & 1.489 & 0.505 & 2.938 & 1.378 \\
\hline Co (nsp) & 5.028 & 2.302 & 5.902 & 2.379 & 0.975 & 5.619 & 2.366 & 0.821 \\
\hline Co (sp) & 5.463 & 2.355 & 6.178 & 2.441 & 0.974 & 5.901 & 2.397 & 0.874 \\
\hline Cr (nsp) & 6.440 & 2.340 & 7.388 & 2.788 & 0.716 & 7.411 & 2.280 & 1.053 \\
\hline Cr (sp) & 6.735 & 2.690 & 7.388 & 2.788 & 0.716 & 7.411 & 2.280 & 1.053 \\
\hline Cs & 0.554 & 5.385 & 0.635 & 5.213 & 2.110 & 0.626 & 5.250 & 2.031 \\
\hline $\mathrm{Cu}$ & 3.154 & 2.428 & 3.407 & 2.496 & 1.063 & 3.280 & 2.468 & 0.912 \\
\hline $\mathrm{Fe}(\mathrm{nsp})$ & 5.780 & 2.287 & 6.903 & 2.400 & 0.927 & 6.496 & 2.377 & 0.800 \\
\hline $\mathrm{Fe}(\mathrm{sp})$ & 6.314 & 2.405 & 6.903 & 2.400 & 0.927 & 6.893 & 2.391 & 0.969 \\
\hline $\mathrm{Ga}$ & 2.261 & 2.749 & 2.516 & 2.822 & 1.298 & 2.497 & 2.683 & 1.225 \\
\hline $\mathrm{Hf}$ & 4.850 & 2.919 & 6.091 & 3.152 & 1.140 & 6.082 & 3.051 & 0.999 \\
\hline $\mathrm{Hg}$ & 0.093 & 3.534 & 0.128 & 3.553 & 1.471 & 0.123 & 3.574 & 1.249 \\
\hline In & 1.912 & 3.162 & 2.165 & 3.226 & 1.459 & 2.136 & 3.069 & 1.382 \\
\hline Ir & 6.948 & 2.564 & 7.739 & 2.662 & 1.101 & 7.312 & 2.663 & 0.908 \\
\hline K & 0.700 & 4.527 & 0.810 & 4.636 & 1.901 & 0.787 & 4.430 & 1.763 \\
\hline $\mathrm{Li}$ & 1.311 & 3.091 & 1.561 & 3.102 & 1.159 & 1.538 & 2.960 & 1.085 \\
\hline $\mathrm{Lu}$ & 2.595 & 3.338 & 3.533 & 3.433 & 1.320 & 3.434 & 3.419 & 1.103 \\
\hline $\mathrm{Mg}$ & 0.952 & 3.058 & 1.241 & 3.087 & 1.354 & 1.106 & 3.046 & 1.174 \\
\hline Mn (nsp) & 6.322 & 2.296 & 7.437 & 2.447 & 0.921 & 7.119 & 2.618 & 0.604 \\
\hline Mn (sp) & 6.871 & 2.573 & 7.437 & 2.447 & 0.921 & 7.119 & 2.618 & 0.604 \\
\hline Mo & 7.931 & 2.597 & 8.879 & 3.102 & 0.808 & 8.907 & 2.553 & 1.138 \\
\hline $\mathrm{Na}$ & 0.937 & 3.659 & 1.064 & 3.681 & 1.519 & 1.036 & 3.519 & 1.404 \\
\hline $\mathrm{Nb}$ & 7.122 & 2.700 & 7.865 & 3.187 & 0.907 & 8.157 & 2.663 & 1.179 \\
\hline $\mathrm{Ni}(\mathrm{nsp})$ & 4.164 & 2.338 & 4.769 & 2.408 & 1.020 & 4.573 & 2.377 & 0.874 \\
\hline Ni (sp) & 4.253 & 2.355 & 4.827 & 2.425 & 1.010 & 4.614 & 2.397 & 0.867 \\
\hline Os & 8.202 & 2.553 & 9.510 & 2.681 & 1.034 & 8.861 & 2.698 & 0.850 \\
\hline $\mathrm{Pb}$ & 2.795 & 3.305 & 3.139 & 3.535 & 1.322 & 3.129 & 3.428 & 1.195 \\
\hline $\mathrm{Pd}$ & 2.847 & 2.631 & 3.407 & 2.701 & 1.175 & 3.238 & 2.658 & 1.024 \\
\hline $\mathrm{Pt}$ & 5.082 & 2.611 & 5.391 & 2.695 & 1.205 & 5.150 & 2.650 & 1.040 \\
\hline $\mathrm{Rb}$ & 0.619 & 4.925 & 0.715 & 4.974 & 2.051 & 0.692 & 4.754 & 1.903 \\
\hline $\mathrm{Re}$ & 8.633 & 2.573 & 10.057 & 2.724 & 1.033 & 9.498 & 2.944 & 0.672 \\
\hline $\mathrm{Rh}$ & 4.643 & 2.551 & 5.569 & 2.634 & 1.092 & 5.265 & 2.620 & 0.923 \\
\hline $\mathrm{Ru}$ & 6.321 & 2.528 & 7.592 & 2.663 & 1.015 & 7.147 & 2.668 & 0.845 \\
\hline $\mathrm{Sc}$ & 2.706 & 3.149 & 3.619 & 3.283 & 1.199 & 3.553 & 3.218 & 1.038 \\
\hline Sn & 3.010 & 3.137 & 3.353 & 3.315 & 1.300 & 3.339 & 3.196 & 1.195 \\
\hline $\mathrm{Sr}$ & 0.966 & 4.249 & 1.332 & 4.287 & 1.653 & 1.279 & 4.138 & 1.480 \\
\hline $\mathrm{Ta}$ & 6.963 & 2.729 & 8.102 & 2.811 & 1.266 & 8.089 & 2.712 & 1.121 \\
\hline Tc & 7.583 & 2.538 & 8.931 & 2.710 & 1.008 & 8.530 & 2.894 & 0.677 \\
\hline $\mathrm{Ti}$ & 4.450 & 2.674 & 5.469 & 2.933 & 1.030 & 5.536 & 2.795 & 0.930 \\
\hline $\mathrm{Tl}$ & 1.649 & 3.318 & 1.849 & 3.375 & 1.527 & 1.819 & 3.214 & 1.467 \\
\hline $\mathrm{V}$ & 5.845 & 2.445 & 6.654 & 2.885 & 0.806 & 6.874 & 2.401 & 1.067 \\
\hline W & 8.212 & 2.632 & 9.157 & 3.089 & 0.850 & 9.223 & 2.602 & 1.117 \\
\hline $\mathrm{Y}$ & 2.779 & 3.424 & 3.672 & 3.567 & 1.346 & 3.590 & 3.493 & 1.168 \\
\hline $\mathrm{Zn}$ & 0.862 & 2.538 & 1.039 & 2.569 & 1.308 & 0.817 & 2.537 & 1.194 \\
\hline $\mathrm{Zr}$ & 5.096 & 2.918 & 6.059 & 3.203 & 1.166 & 6.112 & 3.016 & 1.077 \\
\hline
\end{tabular}




\section{Group 1: Li, Na, K, Rb, and Cs (alkali metals)}

Figures 7, 8, 9, 10, and 11 show the phonon band structures of $\mathrm{Li}, \mathrm{Na}, \mathrm{K}, \mathrm{Rb}$, and $\mathrm{Cs}$, respectively. The HX Rb is dynamically stable, while $\mathrm{HX} \mathrm{Li}, \mathrm{Na}, \mathrm{K}$, and Cs are unstable, since the imaginary frequencies in the ZA branch are observed around $\Gamma$. When the bHC phase is assumed, the ZA phonon modes are stabilized. This yields the dynamical stability of $2 \mathrm{D} \mathrm{Li}, \mathrm{Na}$, and $\mathrm{K}$, while the bHC Cs is still unstable. The alkali metals in the bSQ structure are all unstable.
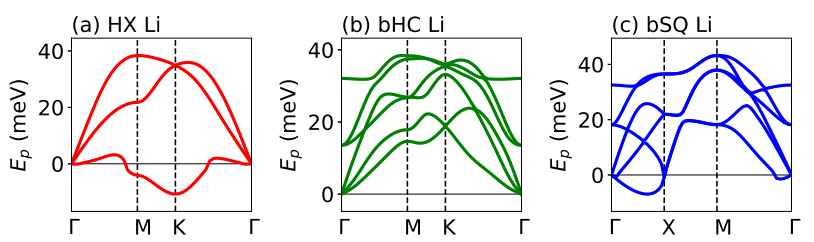

FIG. 7: The phonon band structure of 2D Li for (a) HX, (b) bHC, and (c) bSQ structures.
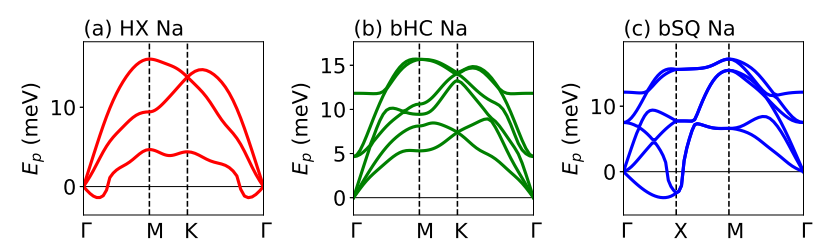

FIG. 8: Same as Fig. 7 but for Na.
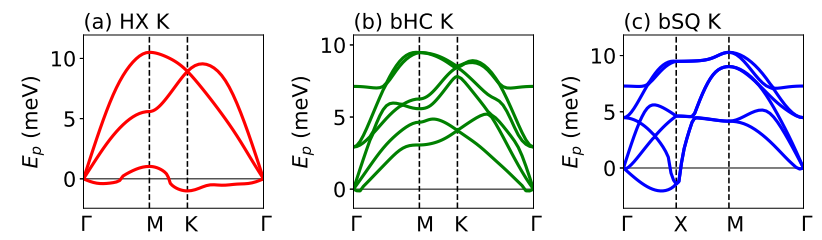

FIG. 9: Same as Fig. 7 but for K.
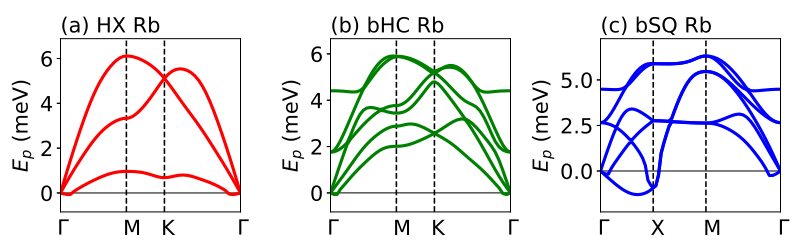

FIG. 10: Same as Fig. 7 but for Rb.
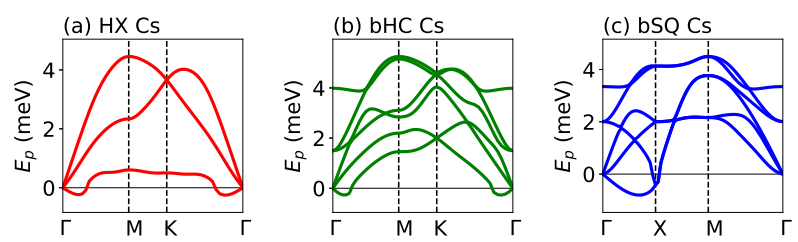

FIG. 11: Same as Fig. 7 but for Cs.

\section{Group 2: $\mathrm{Be}, \mathrm{Mg}, \mathrm{Ca}, \mathrm{Sr}$, and $\mathrm{Ba}$ (alkali earth metals)}

Figures $12,13,14,15$, and 16 show the phonon band structures of $\mathrm{Be}, \mathrm{Mg}, \mathrm{Ca}, \mathrm{Sr}$, and $\mathrm{Ba}$, respectively. The $\mathrm{HX}$ and bHC structures are dynamically stable except $\mathrm{Ba}$. As the ion mass increases in the HX structure, the phonon softening behavior is observed in ZA branch. The maximum phonon energy is higher than that of the alkali metal in the same period because as listed in Table I the values of $E_{\mathrm{c}, j}$ and $a_{j}$ in the alkali earth metals are larger and smaller than those in the alkali metals, respectively. The bSQ structure is dynamically unstable in alkali earth metals. The band structures of bHC and bSQ Ba are omitted due to no convergence in the scf calculations.
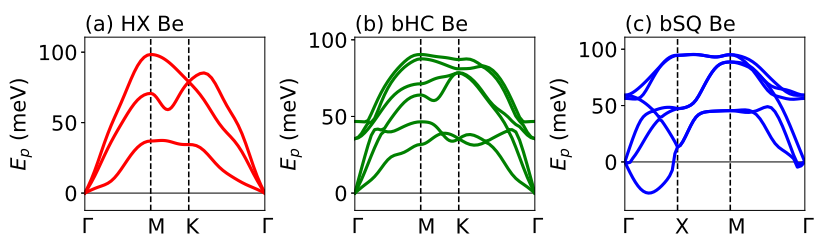

FIG. 12: Same as Fig. 7 but for Be.
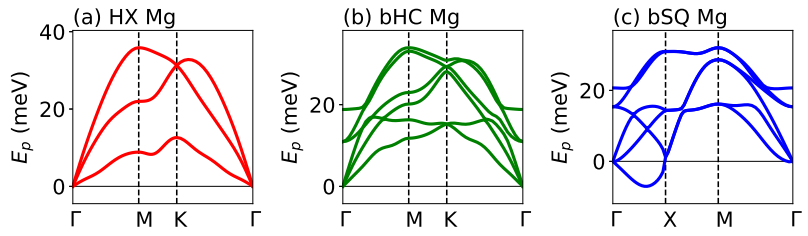

FIG. 13: Same as Fig. 7 but for $\mathrm{Mg}$.
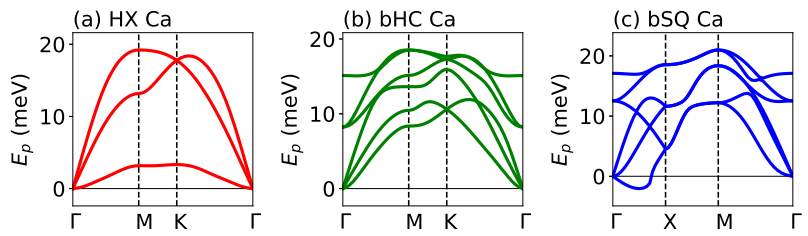

FIG. 14: Same as Fig. 7 but for Ca. 

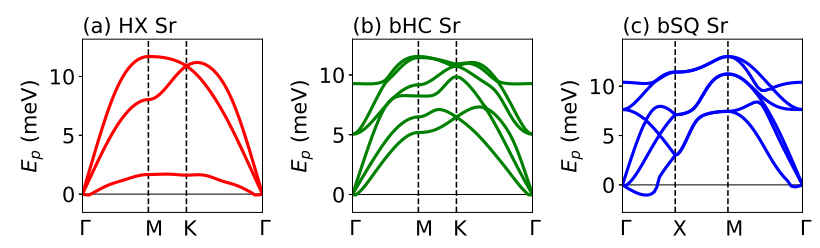

FIG. 15: Same as Fig. 7 but for Sr.
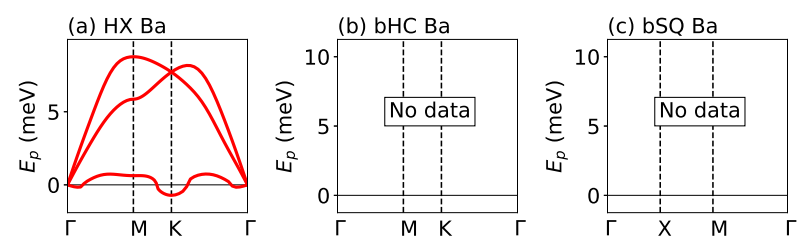

FIG. 16: Same as Fig. 7 but for Ba.

\section{Group 3: Sc, Y, and Lu}

Figures 17, 18, and 19 show the phonon band structures of Sc, Y, and Lu, respectively. The bHC and bSQ structures are dynamically stable. Since the bHC is energetically more stable than the bSQ structure as listed in Table I, the bSQ is regarded as a metastable state.
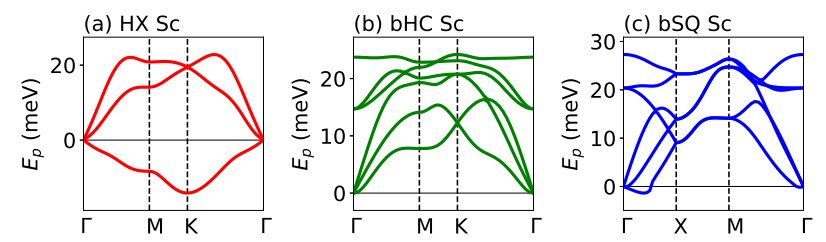

FIG. 17: Same as Fig. 7 but for Sc.
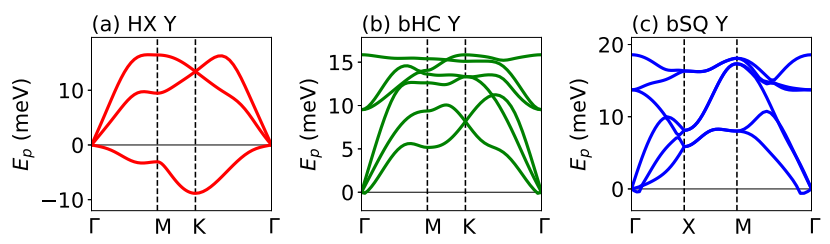

FIG. 18: Same as Fig. 7 but for Y.
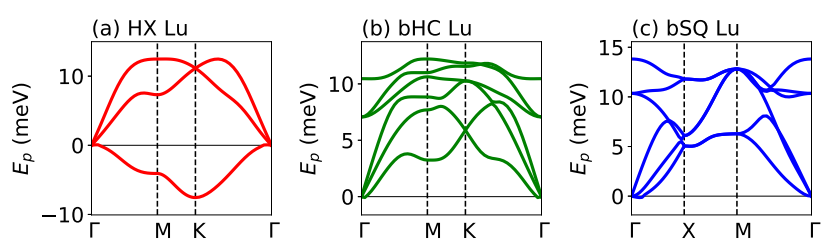

FIG. 19: Same as Fig. 7 but for Lu.

\section{Group 4: $\mathrm{Ti}, \mathrm{Zr}$, and $\mathrm{Hf}$}

Figures 20, 21, and 22 show the phonon band structures of $\mathrm{Ti}, \mathrm{Zr}$, and Hf, respectively. The bSQ structure is dynamically stable for these elements. For Hf, the bHC is energetically more stable than the bSQ structure by 9 $\mathrm{meV}$ per unit cell, which gives the dynamical stability in the bHC Hf.
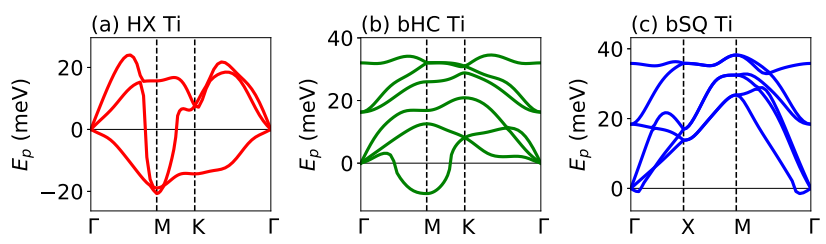

FIG. 20: Same as Fig. 7 but for Ti.
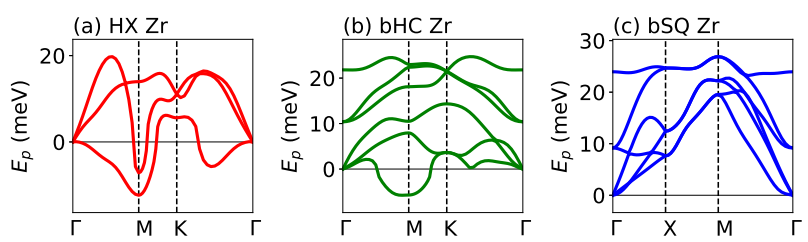

FIG. 21: Same as Fig. 7 but for Zr.
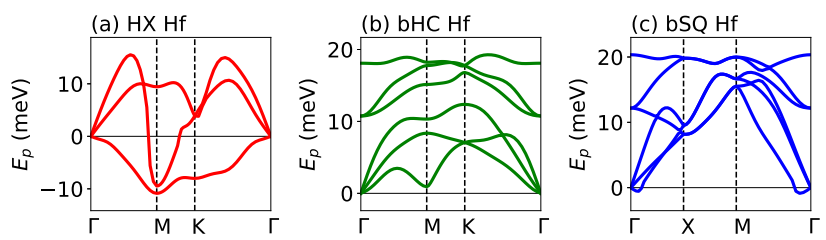

FIG. 22: Same as Fig. 7 but for Hf.

\section{Group 5: V, Nb, and Ta}

Figures 23,24 , and 25 show the phonon band structures of $\mathrm{V}, \mathrm{Nb}$, and $\mathrm{Ta}$, respectively. No stable structures are obtained. As shown later, the 3SQ structure is dynamically stable.
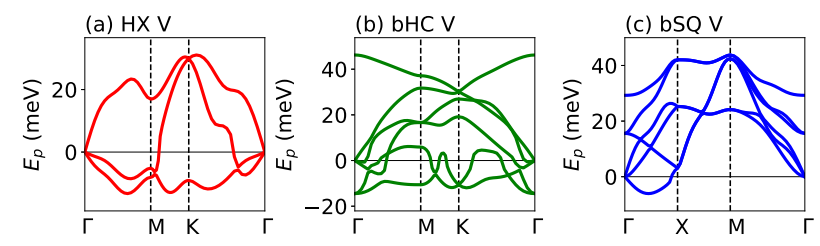

FIG. 23: Same as Fig. 7 but for V. 

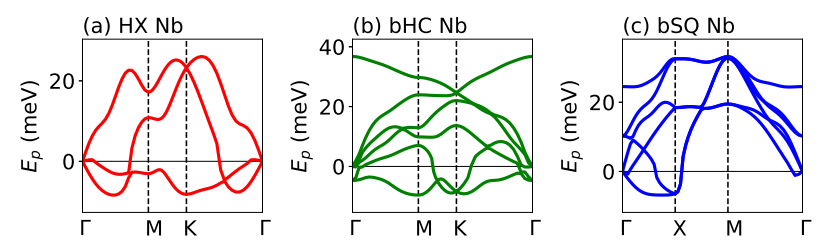

FIG. 24: Same as Fig. 7 but for Nb.
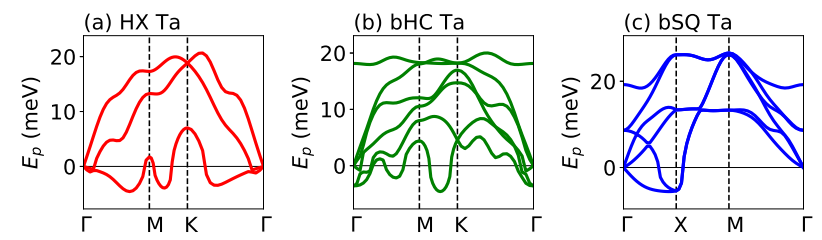

FIG. 25: Same as Fig. 7 but for Ta.

\section{Group 6: Cr, Mo, and W}

Figures 26, 27, and 28 show the phonon band structures of $\mathrm{Cr}$, Mo, and $\mathrm{W}$, respectively. These elements in the bHC structure are dynamically stable. It should be noted that for Mo and W, we have found other bHC structure different from the bHC structure listed in Table I. Although the former has a larger $E_{\mathrm{c}, 2}$, smaller $a_{2}$, and higher buckling height $\delta_{2}$, such a structure is dynamically unstable.
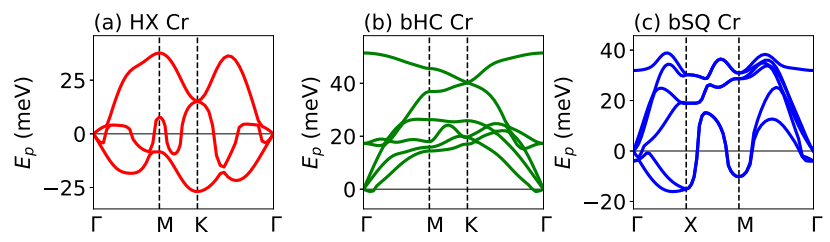

FIG. 26: Same as Fig. 7 but for Cr.
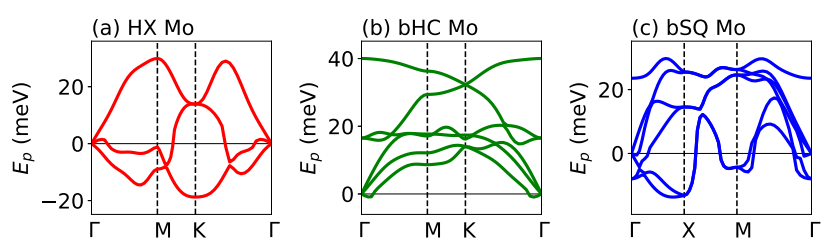

FIG. 27: Same as Fig. 7 but for Mo.
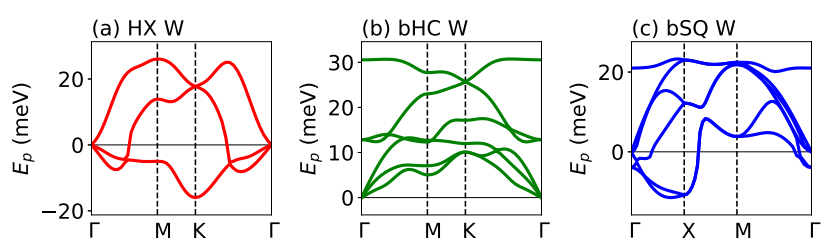

FIG. 28: Same as Fig. 7 but for W.

\section{Group 7: Mn, Tc, and Re}

Figures 29, 30, and 31 show the phonon band structures of $\mathrm{Mn}, \mathrm{Tc}$, and Re, respectively. The bHC and bSQ structures are dynamically stable in these elements, whereas the bSQ structure is metastable.
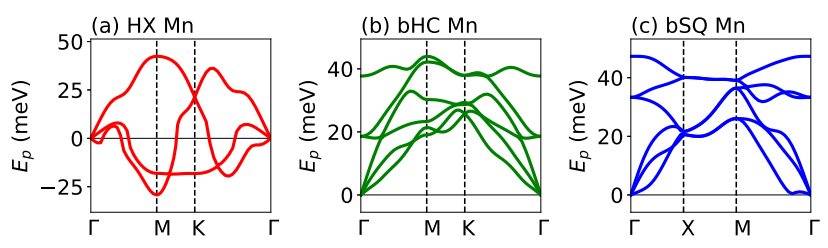

FIG. 29: Same as Fig. 7 but for Mn.
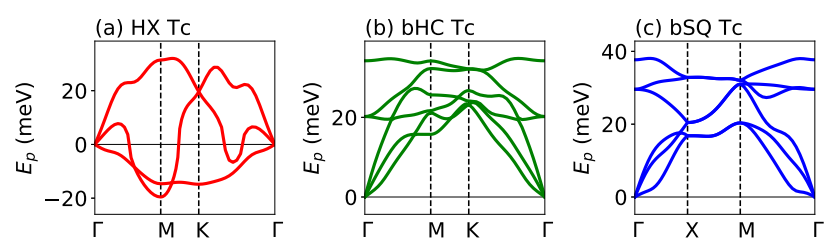

FIG. 30: Same as Fig. 7 but for Tc.
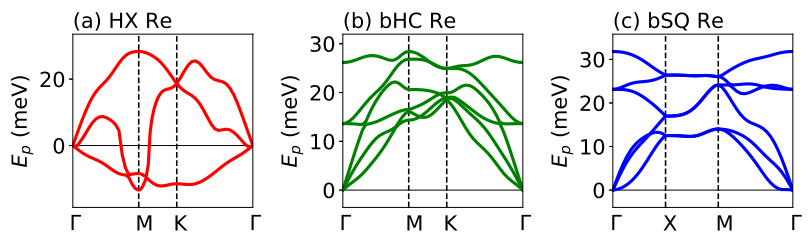

FIG. 31: Same as Fig. 7 but for Re.

\section{Group 8: Fe, Ru, and Os}

Figures 32,33 , and 34 show the phonon band structures of $\mathrm{Fe}, \mathrm{Ru}$, and $\mathrm{Os}$, respectively. These have the bHC and bSQ as dynamically stable structures, while the bSQ is a metastable structure.
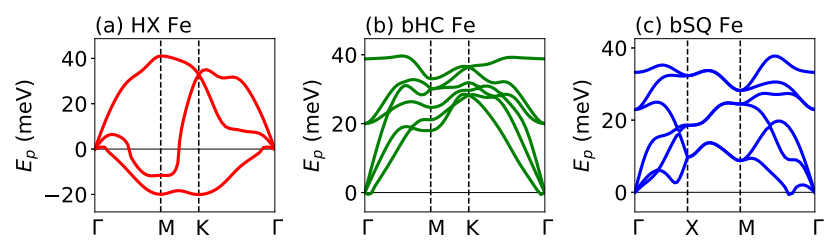

FIG. 32: Same as Fig. 7 but for Fe. 

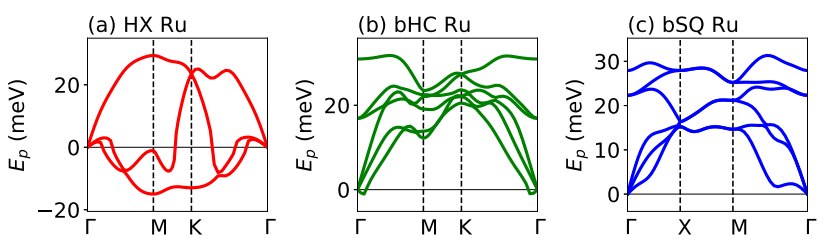

FIG. 33: Same as Fig. 7 but for Ru.
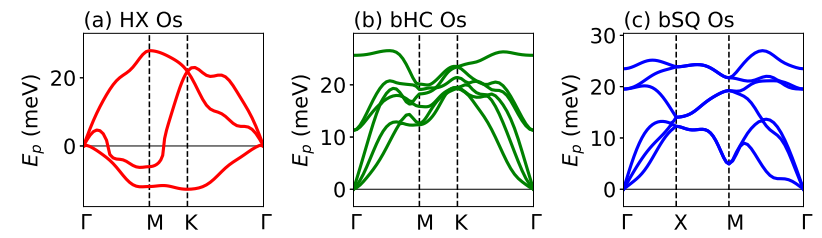

FIG. 34: Same as Fig. 7 but for Os.

\section{Group 9: Co, Rh, and Ir}

Figures 35, 36, and 37 show the phonon band structures of Co, Rh, and Ir, respectively. These elements in the bHC and bSQ structures are dynamically stable, while the bSQ structure is metastable.
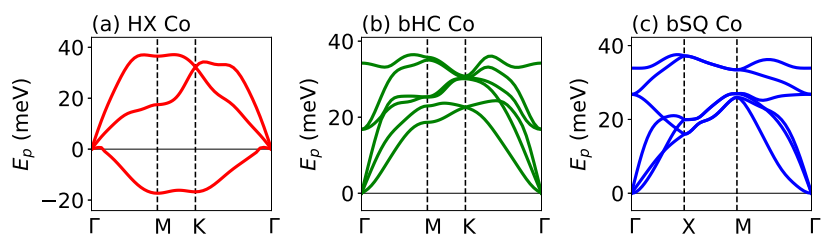

FIG. 35: Same as Fig. 7 but for Co.
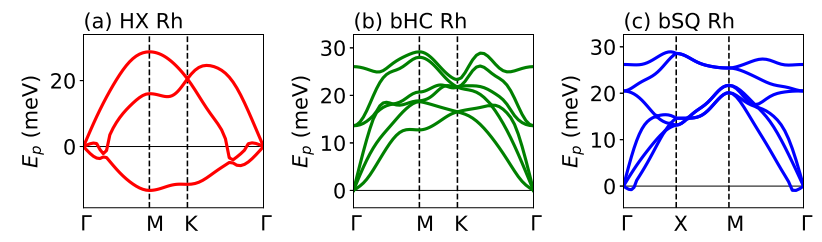

FIG. 36: Same as Fig. 7 but for Rh.
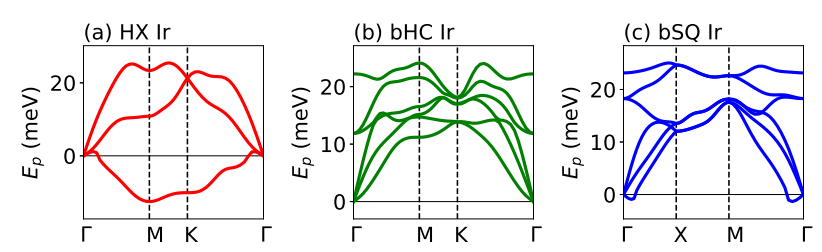

FIG. 37: Same as Fig. 7 but for Ir.

\section{Group 10: Ni, Pd, and Pt}

Figures 38, 39, and 40 show the phonon band structures of $\mathrm{Ni}, \mathrm{Pd}$, and $\mathrm{Pt}$, respectively. These elements in the bHC and bSQ structures are dynamically stable, while the bSQ is a metastable structure.
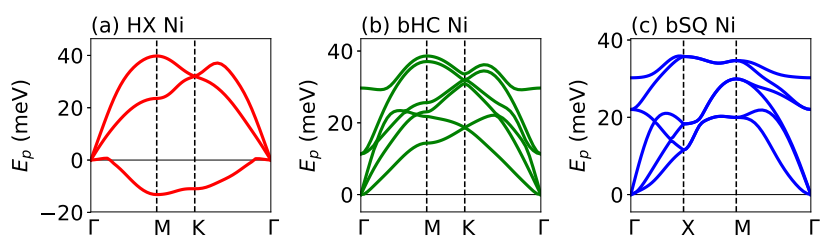

FIG. 38: Same as Fig. 7 but for Ni.
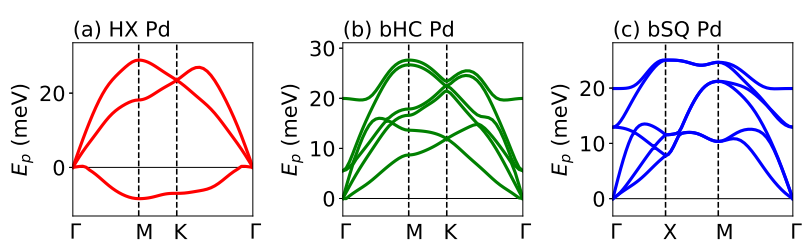

FIG. 39: Same as Fig. 7 but for Pd.
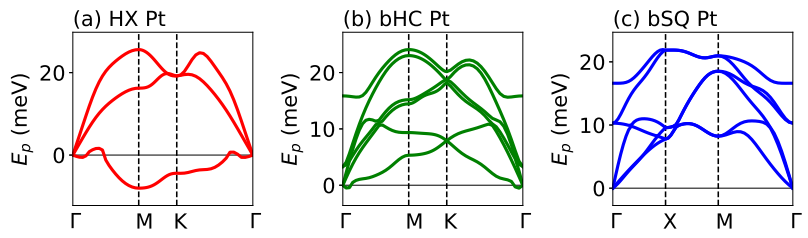

FIG. 40: Same as Fig. 7 but for Pt.

\section{Group 11: Cu, Ag, and Au (nobel metals)}

Figures 41, 42 (the same as in Fig. 3 in the main text), and 43 show the phonon band structure of $\mathrm{Cu}, \mathrm{Ag}$, and $\mathrm{Au}$, respectively. The HX and $\mathrm{bHC}$ structures are dynamically stable. The former result (the stability of $\mathrm{HX}$ ) is consistent with the previous calculations $[11,12,13] . \mathrm{Cu}$ is dynamically stable in the form of bSQ structure and is the only element with no imaginary frequencies observed.
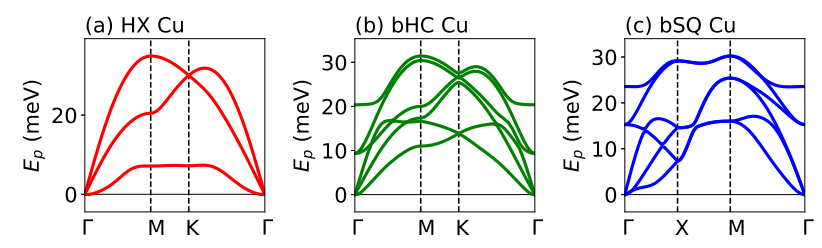

FIG. 41: Same as Fig. 7 but for $\mathrm{Cu}$. 

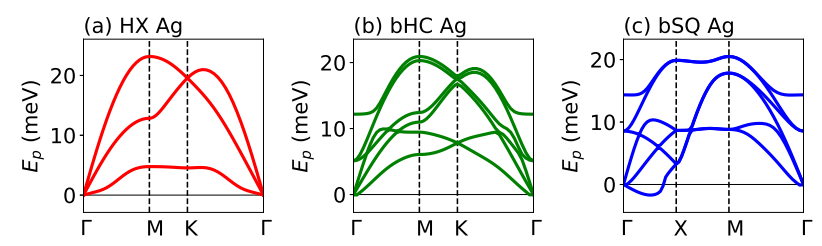

FIG. 42: Same as Fig. 7 but for Ag.
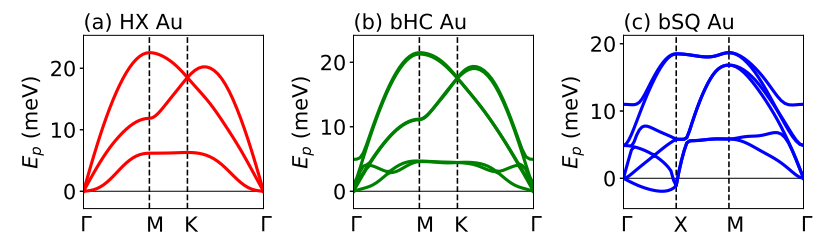

FIG. 43: Same as Fig. 7 but for Au.

\section{Group 12: Zn, Cd, and $\mathrm{Hg}$}

Figures 44, 45, and 46 show the phonon band structures of $\mathrm{Zn}, \mathrm{Cd}$, and $\mathrm{Hg}$, respectively. For $\mathrm{Zn}$ and $\mathrm{Cd}$, the HX and bHC structures are dynamically stable, while for $\mathrm{Hg}$ the bSQ structure is dynamically stable.
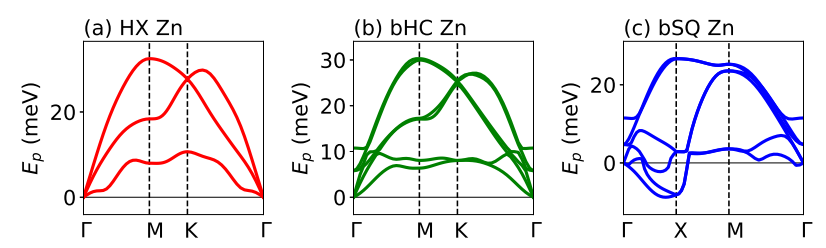

FIG. 44: Same as Fig. 7 but for $\mathrm{Zn}$.
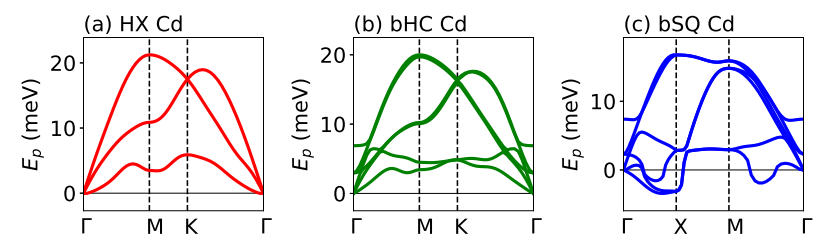

FIG. 45: Same as Fig. 7 but for Cd.
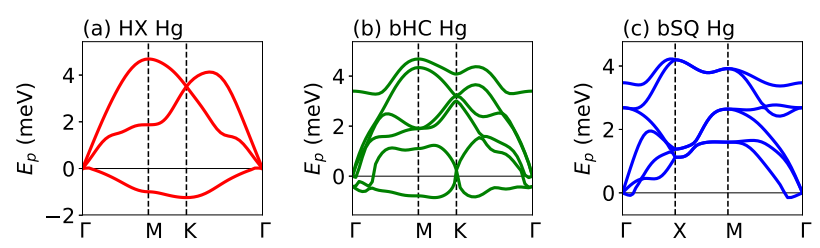

FIG. 46: Same as Fig. 7 but for $\mathrm{Hg}$.

\section{Group 13: Al, Ga, In, and Tl}

Figures $47,48,49$, and 50 show the phonon band structure of $\mathrm{Al}, \mathrm{Ga}, \mathrm{In}$, and $\mathrm{Tl}$, respectively. For all the elements, the HX structure is unstable. The bHC and bSQ structures are dynamically stable in $\mathrm{Al}$ only. The stability of the trilayer structures will be investigated for Ga, In, and $\mathrm{Tl}$, below.
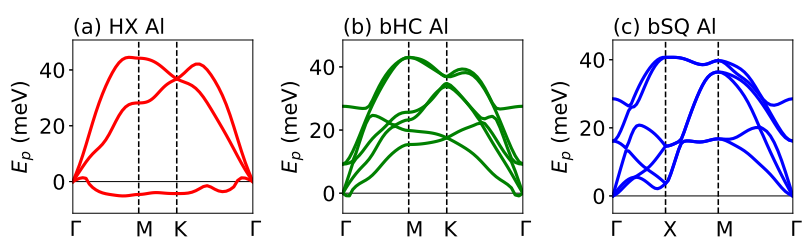

FIG. 47: Same as Fig. 7 but for $\mathrm{Al}$.
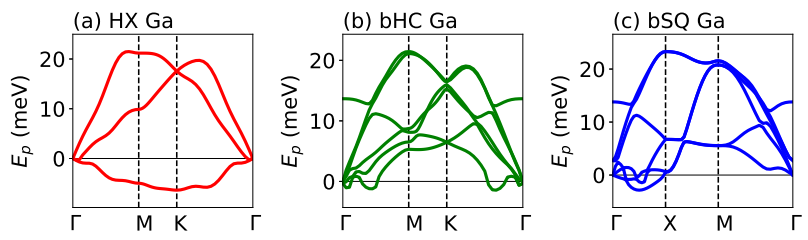

FIG. 48: Same as Fig. 7 but for Ga.
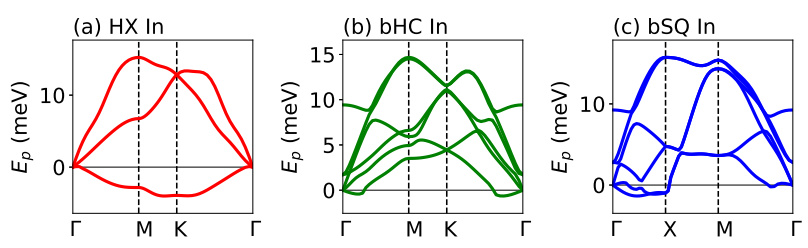

FIG. 49: Same as Fig. 7 but for In.
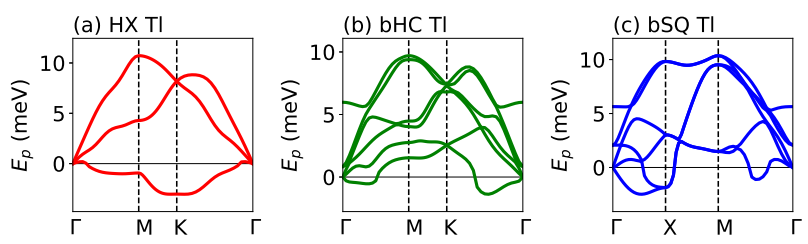

FIG. 50: Same as Fig. 7 but for Tl.

\section{Group 14: Sn and $\mathrm{Pb}$}

Figures 51 and 52 show the phonon band structure of $\mathrm{Sn}$ and $\mathrm{Pb}$, respectively. $\mathrm{Sn}$ and $\mathrm{Pb}$ have the $\mathrm{bHC}$ as a dynamically stable structure. The bSQ Pb is a metastable phase. 

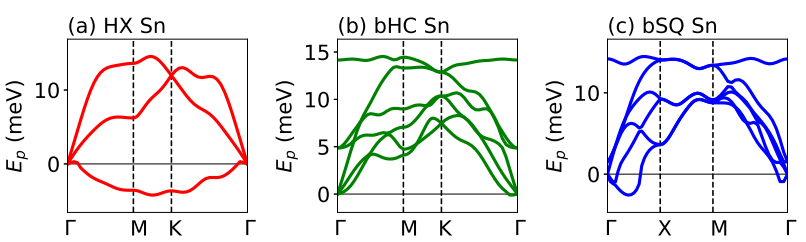

FIG. 51: Same as Fig. 7 but for Sn.
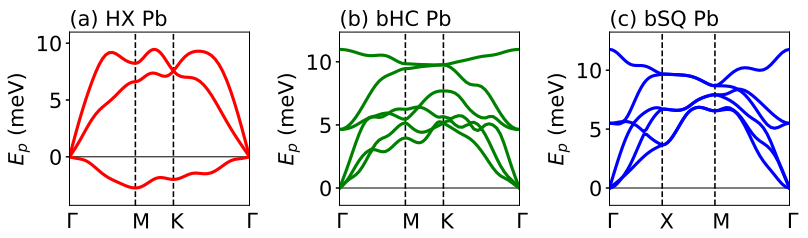

FIG. 52: Same as Fig. 7 but for $\mathrm{Pb}$.

\section{Spin-polarized Co and Ni}

Figures 53(a) and 53(b) show the phonon band structures of bHC $\mathrm{Co}$ and $\mathrm{bHC} \mathrm{Ni}$, respectively. Both are dynamically stable.
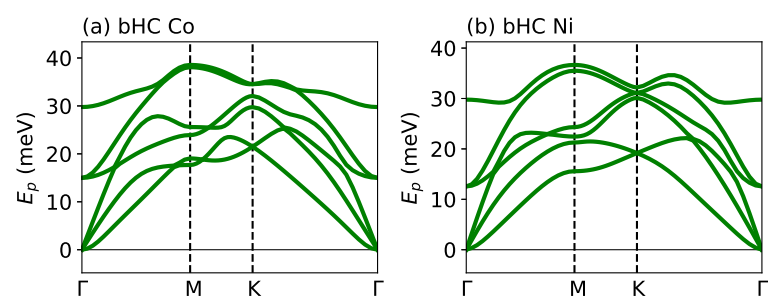

FIG. 53: The phonon band structures of (a) bHC Co and (b) bHC Ni. Spin-polarized calculations are performed.

\section{Trilayers}

Figures 54 and 55 show the phonon band structures of group 5 metals (V, Nb, and $\mathrm{Ta}$ ) and group 13 metals (Ga, In, and $\mathrm{Tl}$ ), respectively: Left for $3 \mathrm{HX}$ and right for 3SQ structures. For group 5 metals, the 3SQ structure is dynamically stable, while the $3 \mathrm{HX}$ structure is unstable. For group 13 metals, the $3 \mathrm{HX}$ In is dynamically stable only.
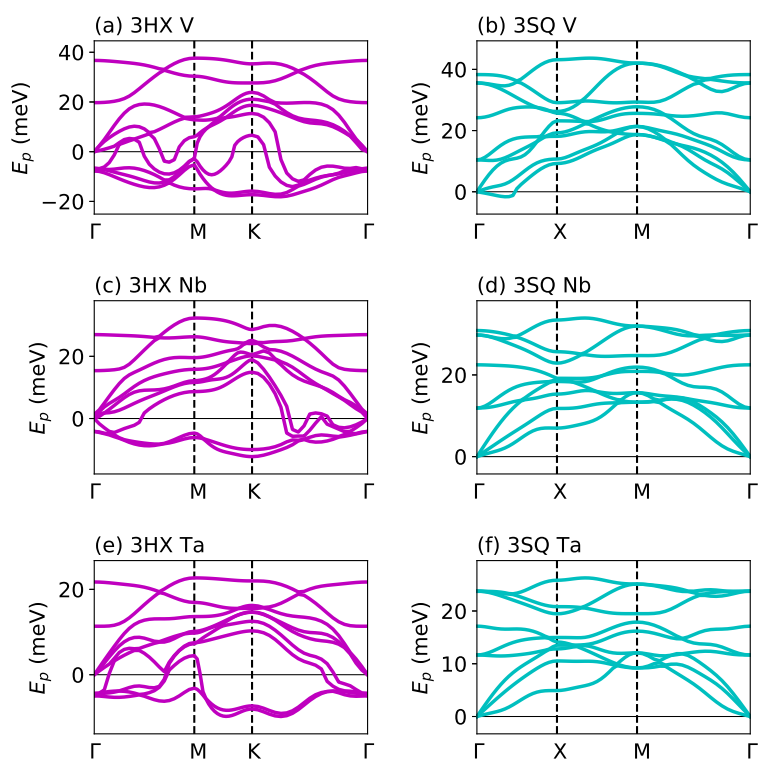

FIG. 54: The phonon band structures of group 5 metals of $\mathrm{V}, \mathrm{Nb}$, and Ta: Left for $3 \mathrm{HX}$ and right for 3SQ structures.
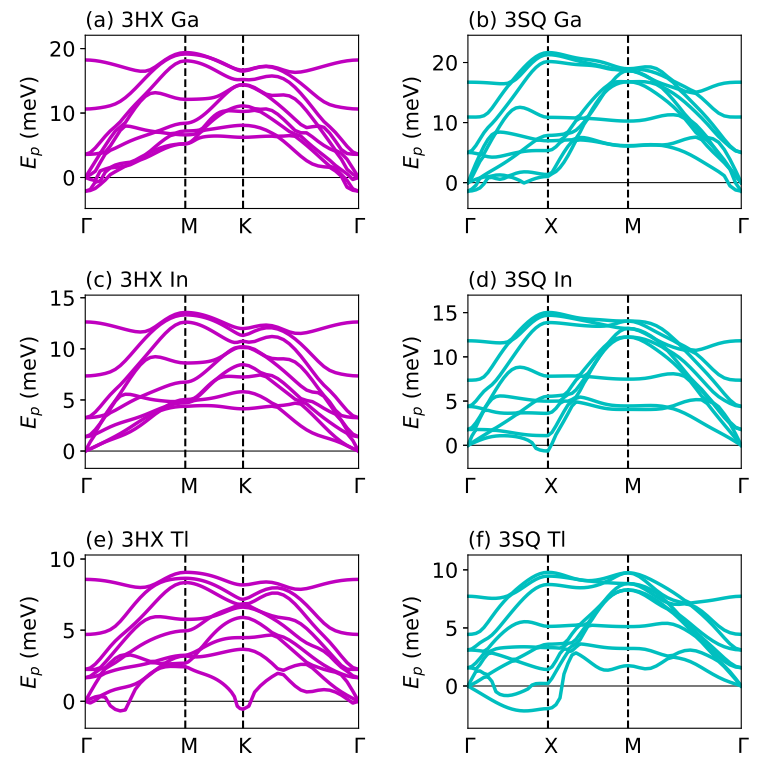

FIG. 55: Same as Fig. 54 but for group 13 metals of Ga, In, and $\mathrm{Tl}$. 


\section{D alloys}

Figure 56 shows the time-evolution of the displacement vector $\boldsymbol{u}=\left(u_{x}, u_{y}, u_{z}\right)$ from the equilibrium position of an $\mathrm{Al}$ atom in a unit cell of $\mathrm{B}_{h} \mathrm{AlCu}$ at $T=2500 \mathrm{~K}$ : The oscillation amplitude is about $0.4 \AA$ that is smaller than the lattice constant $a=2.787 \AA$. Above $T=3000 \mathrm{~K}$, no scf convergence is obtained after some MD steps, since the atoms deviate from the equilibrium position largely.

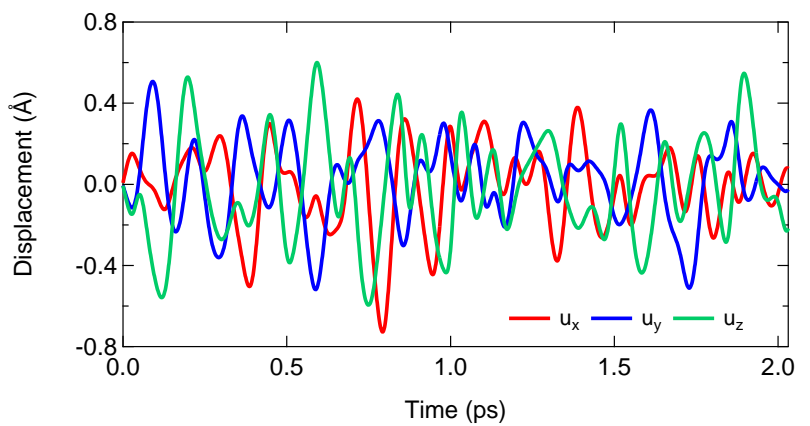

FIG. 56: The time-evolution of $\boldsymbol{u}$ for an $\mathrm{Al}$ atom in a unit cell of $\mathrm{B}_{h} \mathrm{AlCu}$.
Figure 57 shows the phonon band structure of $\mathrm{B}_{h}$ CuZn. No imaginary phonon frequencies are observed, indicating that $\mathrm{B}_{h} \mathrm{CuZn}$ is dynamically stable at $T=0$ $\mathrm{K}$. However, no scf convergence is obtained during the MD calculations assuming $2 \times 2 \times 2$ supercell.

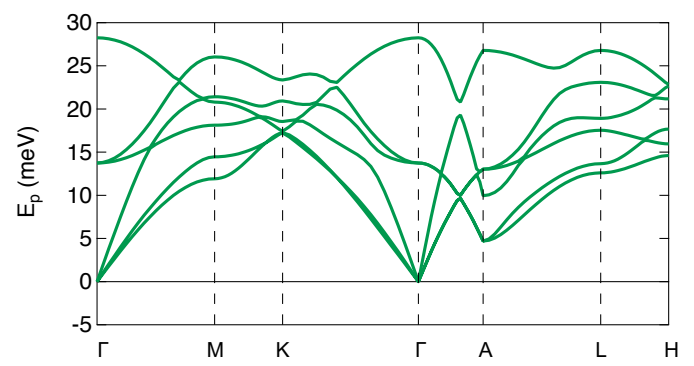

FIG. 57: The phonon band structure of $\mathrm{B}_{h} \mathrm{CuZn}$.
[1] N. W. Ashcroft, N. D. Mermin, and D. Wei, Solid State Physics, revised edition (Cengage Learning, Singapore, 2016).

[2] G. Grimvall, B. Magyari-Köpe, V. Ozoliņš, and K. A. Persson, Lattice instabilities in metallic elements, Rev. Mod. Phys. 84, 945 (2012).

[3] A. Togo and I. Tanaka, Evolution of crystal structures in metallic elements, Phys. Rev. B 87, 184104 (2013).

[4] S. Schönecker, X. Li, M. Richter, and L. Vitos, Lattice dynamics and metastability of FCC metals in the HCP structure and the crucial role of spin-orbit coupling in platinum, Phys. Rev. B 97, 224305 (2018).

[5] X. Huang, S. Li, Y. Huang, S. Wu, X. Zhou, S. Li, C. L. Gan, F. Boey, C. A. Mirkin, and H. Zhang, Synthesis of hexagonal close-packed gold nanostructures, Nat. Commun. 2, 292 (2011).

[6] A.-X. Yin, W.-C. Liu, J. Ke, W. Zhu, J. Gu, Y.-W. Zhang, and C.-H. Yan, Ru Nanocrystals with ShapeDependent Surface-Enhanced Raman Spectra and Catalytic Properties: Controlled Synthesis and DFT Calculations, J. Am. Chem. Soc., 134, 20479 (2012).

[7] X. Kong, K. Xu, C. Zhang, J. Dai, S. N. Oliaee, L. $\mathrm{Li}, \mathrm{X}$. Zeng, C. Wu and Z. Peng, Free-Standing TwoDimensional $\mathrm{Ru}$ Nanosheets with High Activity toward Water Splitting, ACS Catal., 6, 1487 (2016).

[8] K. S. Novoselov, A. K. Geim, S. Morozov, D. Jiang, Y. Zhang, S. Dubonos, I. Grigorieva, and A. Firsov, Electric field effect in atomically thin carbon films, Science 306, 666 (2004).

[9] S. Cahangirov, M. Topsakal, E. Aktürk, H. Şahin, and S. Ciraci, Two- and One-Dimensional Honeycomb Structures of Silicon and Germanium, Phys. Rev. Lett. 102, 236804 (2009).
[10] S. Balendhran, S. Walia, H. Nili, S. Sriram, and M. Bhaskaran, Elemental Analogues of Graphene: Silicene, Germanene, Stanene, and Phosphorene, Small 11, 640 (2015).

[11] L.-M. Yang, T. Frauenheim, and E. Ganz, Properties of the Free-Standing Two-Dimensional Copper Monolayer, J. Nanomater. 2016, 8429510 (2016).

[12] L.-M. Yang, T. Frauenheim, and E. Ganz, The new dimension of silver, Phys. Chem. Chem. Phys. 17, 19695 (2015).

[13] L.-M. Yang, T. Frauenheim, and E. Ganz, Glitter in a 2D monolayer, Phys. Chem. Chem. Phys. 17, 26036 (2015).

[14] B. Feng, O. Sugino, R. Y. Liu, J. Zhang, R. Yukawa, M. Kawamura, T. Iimori, H. Kim, Y. Hasegawa, H. Li, L. Chen, K. Wu, H. Kumigashira, F. Komori, T. C. Chiang, S. Meng, and I. Matsuda, Dirac Fermions in Borophene, Phys. Rev. Lett. 118, 096401 (2017).

[15] W. B. Li, L. J. Kong, C. Y. Chen, J. Gou, S. X. Sheng, W. F. Zhang, H. Li, L. Chen, P. Cheng, and K. H. Wu, Experimental realization of honeycomb borophene, Sci. Bull. 63, 282 (2018).

[16] V. Kochat, A. Samanta, Y. Zhang, S. Bhowmick, P. Manimunda, S. A. S. Asif, A. S. Stender, R. Vajtai, A. K. Singh, C. S. Tiwary, P. M. Ajayan, Atomically thin gallium layers from solid-melt exfoliation. Sci. Adv. 4, e1701373 (2018).

[17] H. Liu, A. T. Neal, Z. Zhu, Z. Luo, X. Xu, D. Tománek, and P. D. Ye, Phosphorene: An unexplored 2D semiconductor with a high hole mobility, ACS Nano 8, 4033 (2014).

[18] S. Zhang, Z. Yan, Y. Li, Z. Chen, and H. Zeng, Atomically thin arsenene and antimonene: Semimetalsemiconductor and indirect-direct band-gap transitions, 
Angew. Chem., Int. Ed. Engl. 54, 3112 (2015).

[19] E. Aktürk, O. Ü. Aktürk, and S. Ciraci, Single and bilayer bismuthene: Stability at high temperature and mechanical and electronic properties, Phys. Rev. B 94, 014115 (2016).

[20] S. Ono, Two-dimensional square lattice polonium stabilized by the spin-orbit coupling, arXiv:2003.08535.

[21] J. Nevalaita and P. Koskinen, Atlas for the properties of elemental two-dimensional metals, Phys. Rev. B 97, 035411 (2018).

[22] J. Hwang, Y. J. Oh, J. Kim, M. M. Sung, and K. Cho, Atomically thin transition metal layers: Atomic layer stabilization and metal-semiconductor transition, J. Appl. Phys. 123, 154301 (2018).

[23] P. Giannozzi et al., Advanced capabilities for materials modelling with Quantum ESPRESSO, J. Phys.: Condens. Matter 29, 465901 (2017).

[24] C. Kittel, Introduction to Solid State Physics, 8th edition (Wiley, Hoboken, NJ, 2005).

[25] S. Baroni, S. Gironcoli, A. D. Corso, and P. Giannozzi, Phonons and related crystal properties from densityfunctional perturbation theory, Rev. Mod. Phys. 73, 515 (2001).

[26] W. Sun, S. T. Dacek, S. P. Ong, G. Hautier, A. Jain, W. D. Richards, A. C. Gamst, K. A. Persson, G. Ceder, The thermodynamic scale of inorganic crystalline metastability. Sci. Adv. 2, e1600225 (2016).

[27] M. Podgórny and J. Goniakowski, Magnetism of hexagonal 3d transition metals, Phys. Rev. B 42, 6683 (1990).

[28] G. Y. Guo and H. H. Wang, Gradient-corrected density functional calculation of elastic constants of $\mathrm{Fe}, \mathrm{Co}$ and $\mathrm{Ni}$ in BCC, FCC and HCP structures, Chin. J. Phys. 38, 949 (2000).

[29] V. T. Witusiewicz, U. Hecht, S. G. Fries, S. Rex, The Ag-Al-Cu system Part I: Reassessment of the constituent binaries on the basis of new experimental data, J. Alloys Compd. 385, 133 (2004).

[30] X. W. Zhou, D. K. Ward, M. E. Foster, An analytical bond-order potential for the aluminum copper binary system, J. Alloys Compd. 680, 752 (2016).

[31] L. H. Beck and C. S. Smith, Copper-Zinc Constitution Diagram, Redetermined in The Vicinity of the Beta Phase by Means of Quantitative Metallography, Trans. Soc. Min. Metall. Explor. Inc. 194, 1079 (1952).

[32] N. Gaston, B. Paulus, U. Wedig, and M. Jansen, Multiple Minima on the Energy Landscape of Elemental Zinc: A Wave Function Based Ab Initio Study, Phys. Rev. Lett. 100, 226404 (2008).

[33] D. Pettifor, Bonding and Structure of Molecules and
Solids (Oxford University Press, New York, 2002).

[34] J. Nevalaita and P. Koskinen, Beyond ideal twodimensional metals: Edges, vacancies, and polarizabilities, Phys. Rev. B 98, 115433 (2018).

[35] W. A. Diery, E. A. Moujaes, R. W. Nunes, Nature of localized phonon modes of tilt grain boundaries in graphene, Carbon 140, 250 (2018).

[36] O. Hellman, I. A. Abrikosov, and S. I. Simak, Lattice dynamics of anharmonic solids from first principles, Phys. Rev. B 84, 180301(R) (2011).

[37] H. M. Zhang, Y. Sun, W. Li, J. P. Peng, C. L. Song, Y. Xing, Q. Zhang, J. Guan, Z. Li, and Y. Zhao et al., Detection of a Superconducting Phase in a Two-Atom Layer of Hexagonal Ga Film Grown on Semiconducting GaN(0001), Phys. Rev. Lett. 114, 107003 (2015).

[38] Y. Liu, Z. Wang, X. Zhang, C. Liu, Y. Liu, Z. Zhou, J. Wang, Q. Wang, Y. Liu, C. Xi, M. Tian, H. Liu, J. Feng, X. C. Xie, and J. Wang, Interface-Induced Zeeman-Protected Superconductivity in Ultrathin Crystalline Lead Films, Phys. Rev. X 8, 021002 (2018).

[39] Y. Cao, V. Fatemi, S. Fang, K. Watanabe, T. Taniguchi, E. Kaxiras, and P. Jarillo-Herrero, Unconventional superconductivity in magic-angle graphene superlattices, Nature 556, 43 (2018).

[40] M. Yankowitz, S. Chen, H. Polshyn, Y. Zhang, K. Watanabe, T. Taniguchi, D. Graf, A. F. Young, and C. R. Dean, Tuning superconductivity in twisted bilayer graphene, Science 363, 1059 (2019).

[41] D. Hobbs, J. Hafner, and D. Spišák, Understanding the complex metallic element Mn. I. Crystalline and noncollinear magnetic structure of $\alpha$-Mn, Phys. Rev. B 68, 014407 (2003).

[42] J. P. Perdew, K. Burke, and M. Ernzerhof, Generalized Gradient Approximation Made Simple, Phys. Rev. Lett. 77, 3865 (1996)

[43] J. P. Perdew, A. Ruzsinszky, G. I. Csonka, O. A. Vydrov, G. E. Scuseria, L. A. Constantin, X. Zhou, and K. Burke, Restoring the Density-Gradient Expansion for Exchange in Solids and Surfaces, Phys. Rev. Lett. 100, 136406 (2008).

[44] A. Dal Corso, Pseudopotentials periodic table: From H to $\mathrm{Pu}$, Computational Material Science 95, 337 (2014).

[45] H. J. Monkhorst and J. D. Pack, Special points for Brillouin-zone integrations, Phys. Rev. B 13, 5188 (1976).

[46] N. Marzari, D. Vanderbilt, A. De Vita, and M. C. Payne, Thermal Contraction and Disordering of the $\mathrm{Al}(110)$ Surface, Phys. Rev. Lett. 82, 3296 (1999). 